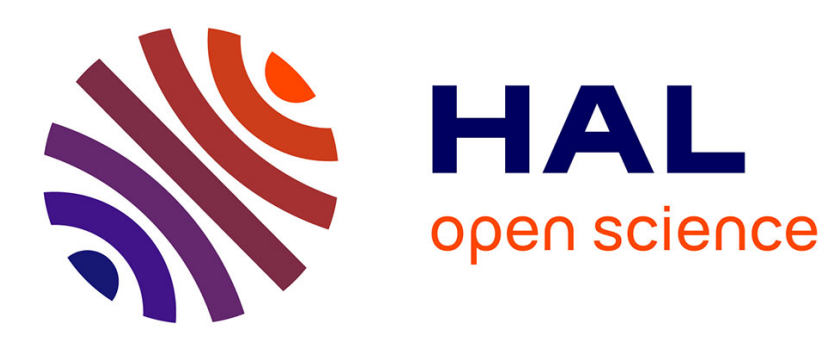

\title{
On existence and approximation for a 3D model of thermally-induced phase transformations in shape-memory alloys
}

\author{
Alexander Mielke, Laetitia Paoli, Adrien Petrov
}

\section{- To cite this version:}

Alexander Mielke, Laetitia Paoli, Adrien Petrov. On existence and approximation for a 3D model of thermally-induced phase transformations in shape-memory alloys. SIAM Journal on Mathematical Analysis, 2009, 41 (4), pp.1388-1414. 10.1137/080726215 . hal-00863759

\section{HAL Id: hal-00863759}

\section{https://hal.science/hal-00863759}

Submitted on 6 Mar 2019

HAL is a multi-disciplinary open access archive for the deposit and dissemination of scientific research documents, whether they are published or not. The documents may come from teaching and research institutions in France or abroad, or from public or private research centers.
L'archive ouverte pluridisciplinaire HAL, est destinée au dépôt et à la diffusion de documents scientifiques de niveau recherche, publiés ou non, émanant des établissements d'enseignement et de recherche français ou étrangers, des laboratoires publics ou privés. 


\title{
ON EXISTENCE AND APPROXIMATION FOR A 3D MODEL OF THERMALLY INDUCED PHASE TRANSFORMATIONS IN SHAPE-MEMORY ALLOYS*
}

\author{
ALEXANDER MIELKE ${ }^{\dagger}$, LAETITIA PAOLI ${ }^{\ddagger}$, AND ADRIEN PETROV $§$
}

\begin{abstract}
This paper deals with a three-dimensional model for thermal stress-induced transformations in shape-memory materials. Microstructure, like twined martensites, is described mesoscopically by a vector of internal variables containing the volume fractions of each phase. We assume that the temperature variations are prescribed. The problem is formulated mathematically within the energetic framework of rate-independent processes. An existence result is proved and temporal regularity is obtained in the case of uniform convexity. We also study space-time discretizations and establish convergence of these approximations.
\end{abstract}

Key words. shape-memory materials, rate-independent energetic formulation, temperatureinduced phase transformation, differential inclusion, convergence for space-time discretization

AMS subject classifications. 49J40, 74C05, 74F05, 74M05, 74N30

DOI. $10.1137 / 080726215$

1. Introduction. The good performances of shape-memory alloys in applications to relative fields like biomedicine, aeronautics, or engineering stimulate the interest in the development of different models. These alloys have some surprising thermomechanical behavior; namely, severely deformed materials can recover their original shape after a thermal cycle (shape-memory effect). In the mathematical literature, many one-dimensional models are available, but multidimensional models allowing for multiaxial loadings and anisotropies are rarely presented. In [MiT99, MTL02] such models were introduced for the isothermal setting and a first existence result was provided.

This paper deals with the quasi-static evolution of shape-memory materials in a small-strain regime under nonisothermal conditions. In [SMZ98, AuS01, AuP04] a model for polycrystalline shape-memory materials is proposed where phase transformations are driven by stress or temperature changes, and it is analyzed in [AuS04, AuS05, MiP07, AMS08]. In this model the mesoscopic average of the transformation strain used an internal variable, and hence it is restricted to situations where isotropy and equal elastic constants in austenite and martensite can be assumed. Here we treat a more advanced model which allows us to describe each pure phase independently, like in the isothermal models considered in [CaP01, MTL02, GMH02, KMR05, RoK06, GHH07].

\footnotetext{
${ }^{\dagger}$ Institut für Mathematik, Humboldt-Universität zu Berlin, Rudower Chaussee 25, 12489 Berlin, Germany (mielke@wias-berlin.de).

¥LaMUSE (Laboratoire de Mathématiques de l’Université de Saint-Etienne), 23 rue Paul Michelon, 42023 Saint-Etienne Cedex 02, France (laetitia.paoli@univ-st-etienne.fr).

$\S$ Weierstraß-Institut für Angewandte Analysis und Stochastik, Mohrenstraße 39, 10117 Berlin, Germany (petrov@wias-berlin.de). This author's work was supported by the Deutsche Forschungsgemeinschaft through the project C18 "Analysis and numerics of multidimensional models for elastic phase transformation in shape-memory alloys" of the Research Center MATHEON.
} 
Following [Mie07, MiP07] we assume here that the temperature $\theta$ is given a priori as an applied load $\theta=\theta_{\text {appl }}(t, x)$. This assumption is used in engineering models and is acceptable if the body is small in at least one direction. Then, the excessive or missing heat can be balanced through the environment. While the existence result will be a direct consequence of the general theory of energetic solutions for rateindependent processes, we present here an approach which states existence of solutions as a consequence of convergence of space-time discretizations. Using the ideas of $\Gamma$-convergence for rate-independent processes developed in [MRS08] we show that for arbitrary sequences of partitions of the time interval and for arbitrary finitedimensional approximations of the underlying Banach space we obtain sequences of discrete solutions that are a priori bounded and precompact. Any limit point of this sequence will be a solution of the full problem. As in general uniqueness of solutions for the full problem is not true, it cannot be expected that the full sequence converges. A similar approach, in a more general setting, is followed in [MiR06].

Our model is based on a stored-energy density $W$ and a dissipation distance $D$. The stored-energy density $W(x, e, z, \theta)$ depends on the material point $x \in \Omega$, the infinitesimal strain $e=\mathbf{e}(u)=\frac{1}{2}\left(\nabla u+\nabla u^{\top}\right)$ for the displacement $u: \Omega \rightarrow \mathbb{R}^{d}$, the prescribed temperature $\theta=\theta_{\text {appl }}(t, x)$, and the vector of phase fractions $z: \Omega \rightarrow Z=$ $\operatorname{conv}\left\{\widehat{e}_{1}, \ldots, \widehat{e}_{N}\right\}$, the convex hull in $\mathbb{R}^{N}$. Here $N$ is the total number of phases; in an austenite-martensite phase transformation this includes the austenite and all variants of martensite. In general, $z \in \operatorname{conv}\left\{\widehat{e}_{1}, \ldots, \widehat{e}_{N}\right\}$ is a phase mixture, and the vertices $z=\widehat{e}_{1}, \ldots, \widehat{e}_{N}$ correspond to the pure phases such that $W\left(\cdot, \widehat{e}_{k}, \cdot, \cdot\right)$ corresponds to the stored-energy density of a pure phase, which can be adapted to measured data. The total stored energy takes the following form:

$$
\mathcal{E}(t, u, z) \stackrel{\text { def }}{=} \int_{\Omega}\left(W\left(x, \mathbf{e}\left(u+u_{\text {Dir }}(t)\right), z, \theta_{\text {appl }}(t)\right)+\frac{\sigma}{2}|\nabla z|^{2}\right) \mathrm{d} x-\langle l(t), u\rangle, \quad \sigma>0,
$$

where $u_{\text {Dir }}$ and $l$ denote the time-dependent Dirichlet boundary data and applied loading, respectively. To model the dissipation via phase transformations we introduce a dissipation distance $D: \Omega \times Z \times Z \rightarrow[0, \infty)$ and define the total dissipation distance $\mathcal{D}$ via

$$
\mathcal{D}\left(z_{0}, z_{1}\right) \stackrel{\text { def }}{=} \int_{\Omega} D\left(x, z_{0}(x), z_{1}(x)\right) \mathrm{d} x
$$

The natural function sets for the unknown $q \stackrel{\text { def }}{=}(u, z)$ is $\mathcal{Q} \stackrel{\text { def }}{=} \mathcal{F} \times \mathcal{Z}$ with $\mathcal{Z} \stackrel{\text { def }}{=}$ $\mathrm{H}^{1}(\Omega ; Z)$. As the time-dependent conditions on $\Gamma_{\text {Dir }} \subset \partial \Omega$ are incorporated in $u_{\text {Dir }}$, we define the space of admissible displacements via

$$
\mathcal{F} \stackrel{\text { def }}{=}\left\{u \in \mathrm{H}^{1}\left(\Omega ; \mathbb{R}^{d}\right) \mid u=0 \text { on } \Gamma_{\text {Dir }}\right\}
$$

Then, our problem can be posed in the energetic formulation for rate-independent problems. For a given initial value $(u(0), z(0)) \in \mathcal{Q}$, we have to find a function $(u, z):[0, T] \rightarrow \mathcal{Q}$ (with $T>0$ ) such that for all $t \in[0, T]$ the global stability condition (S) and the global energy balance (E) are satisfied, i.e.,

(S) $\forall(\bar{u}, \bar{z}) \in \mathcal{Q}: \mathcal{E}(t, u(t), z(t)) \leq \mathcal{E}(t, \bar{u}, \bar{z})+\mathcal{D}(z(t), \bar{z})$

(E) $\mathcal{E}(t, u(t), z(t))+\operatorname{Var}_{\mathcal{D}}(z ;[0, t])=\mathcal{E}(0, u(0), z(0))+\int_{0}^{t} \partial_{s} \mathcal{E}(s, u(s), z(s)) \mathrm{d} s$ 
where the dissipation $\operatorname{Var}_{\mathcal{D}}(z ;[0, t])$ is defined as the supremum over all finite partitions $0 \leq t_{0}<t_{1}<\cdots<t_{n} \leq t$ of $\sum_{j=1}^{n} \mathcal{D}\left(z\left(t_{j-1}\right), z\left(t_{j}\right)\right)$.

The paper is organized as follows. In section 2 , we give a more detailed description of the mechanical model and the mathematical formulation of the problem within the energetic formulation theory of rate-independent systems $(\mathcal{Q}, \mathcal{E}, \mathcal{D})$. In section 3 , we specify the full assumptions and state our existence result by applying the same techniques as in [Mie07, MiP07]. More precisely, we show that for any stable initial data $q(0)$ an energetic solution exists. We also provide a series of further properties of the functional $\mathcal{E}$ that will be used in the later sections.

In section 4 , the temporal smoothness is obtained assuming uniform convexity of $W(x, \cdot, \cdot, \theta)$ and $D\left(x, z_{0}, z_{1}\right)=\psi\left(x, z_{1}-z_{0}\right)$. Finally, in section 5 , we discuss the convergence of space-time discretizations of the problem. For this we choose a sequence $\left(\Pi^{\tau}\right)_{\tau>0}$ of partitions $\left\{0=t_{0}^{\tau}<t_{1}^{\tau}<\cdots<t_{N_{\tau}}^{\tau}=T\right\}$ of the time interval $[0, T]$ with $\max \left\{t_{k}^{\tau}-t_{k-1}^{\tau}: k=1, \ldots, N_{\tau}\right\} \leq \tau$. Moreover, we choose a sequence $\left(\mathcal{Q}_{h}\right)_{h>0}$, $\mathcal{Q}_{h} \stackrel{\text { def }}{=} \mathcal{F}_{h} \times \mathcal{Z}_{h}$, of finite-dimensional space approximations exhausting $\mathcal{Q}$. We obtain a sequence $\bar{q}^{\tau, h}:[0, T] \rightarrow \mathcal{Q}$ of piecewise constant interpolants. The main theorem states that this sequence has a subsequence $\left(\bar{q}^{\tau_{n}, h_{n}}\right)_{n \in \mathbb{N}}$ such that for all $t \in[0, T]$ we have $\bar{q}^{\tau_{n}, h_{n}}(t) \rightarrow q(t)$, where $q:[0, T] \rightarrow \mathcal{Q}$ is a solution for $(\mathcal{Q}, \mathcal{E}, \mathcal{D})$.

In section 6 , we discuss several models for the stored-energy density $W$, which in this context is called mixture function [MiT99, Mie00, CaP01, MTL02] or free energy of mixing [HaG02, GMH02, GHH07]. In particular, we clarify the assumptions that are needed to apply the results obtained in the previous sections.

2. Mechanical model and mathematical formulation. We consider a material with a reference configuration $\Omega \subset \mathbb{R}^{d}$ with $d \in\{2,3\}$. We assume that $\Omega$ is an open bounded set with a 1-regular smooth boundary (see [RaT83]). This body may undergo displacements $u: \Omega \rightarrow \mathbb{R}^{d}$ and phase transformations. The latter will be characterized by a mesoscopic internal variable $z: \Omega \rightarrow Z$, where $Z$ is the Gibbs simplex, associated with the $N$ pure phases $\widehat{e}_{1}, \ldots \widehat{e}_{N} \in \mathbb{R}^{N}$, where $\widehat{e}_{j}$ is the $j$ th unit vector, i.e.,

$$
Z \stackrel{\text { def }}{=} \operatorname{conv}\left\{\widehat{e}_{1}, \ldots, \widehat{e}_{N}\right\} \stackrel{\text { def }}{=}\left\{z=\sum_{i=1}^{N} \lambda_{i} \widehat{e}_{i} \mid 0 \leq \lambda_{i} \leq 1, \sum_{i=1}^{N} \lambda_{i}=1\right\} \subset \mathbb{R}^{N} .
$$

The set of admissible displacements $\mathcal{F}$ is chosen as a suitable subspace of $\mathrm{H}^{1}\left(\Omega ; \mathbb{R}^{d}\right)$ by prescribing Dirichlet data on the subset $\Gamma_{\text {Dir }}$ of $\partial \Omega$, i.e.,

$$
\mathcal{F} \stackrel{\text { def }}{=}\left\{u \in \mathrm{H}^{1}\left(\Omega ; \mathbb{R}^{d}\right)|u|_{\Gamma_{\text {Dir }}}=0\right\} .
$$

Note that the physical displacement is $u+u_{\text {Dir }}$, where $u_{\text {Dir }}:[0, T] \rightarrow \mathrm{H}^{1}\left(\Omega ; \mathbb{R}^{d}\right)$ is prescribed a priori. Throughout the paper we consider the extension of $u_{\text {Dir }}(t)$ to $\Omega$, but actually only the trace on $\Gamma_{\text {Dir }}$ would be needed. The internal variable $z$ belongs to

$$
\mathcal{Z} \stackrel{\text { def }}{=}\left\{z \in \mathrm{H}^{1}\left(\Omega ; \mathbb{R}^{N}\right) \mid z(x) \in Z \text { a.e. } x \in \Omega\right\} \text {. }
$$

We will denote the norm in $\mathcal{Q} \stackrel{\text { def }}{=} \mathcal{F} \times \mathcal{Z}$ by $\|\cdot\|_{\mathcal{Q}}$ and $q \stackrel{\text { def }}{=}(u, z)$.

We also assume that the material behavior depends on the temperature $\theta$, which will be considered as a time-dependent given parameter. Therefore we will not solve an associated heat equation, but we will treat $\theta$ as an applied load and denote it by 
$\theta_{\text {appl }}:[0, T] \times \Omega \rightarrow\left[\theta_{\min }, \theta_{\max }\right]$. This approximation for the temperature is used in engineering models and is justified when the changes of the loading are slow and the body is small in at least one direction: in such a case, excess of heat can be transported very fast to the surface of the body and then radiated into the environment.

We will denote by $\mathbb{R}_{\text {sym }}^{d \times d}$ the space of symmetric $d \times d$ tensors endowed with the scalar product $v: w \stackrel{\text { def }}{=} \operatorname{tr}\left(v^{\top} w\right)$, and the corresponding norm is given by $|v|^{2} \stackrel{\text { def }}{=} v: v$ for all $v, w \in \mathbb{R}_{\mathrm{sym}}^{d \times d}$. Here $(\cdot)^{\top}$ and $\operatorname{tr}(\cdot)$ denote the transpose and the trace of the matrix $(\cdot)$, respectively. The linearized strain tensor is given by $e=\mathbf{e}(u) \stackrel{\text { def }}{=} \frac{1}{2}\left(\nabla u+\nabla u^{\top}\right) \in$ $\mathbb{R}_{\mathrm{sym}}^{d \times d}$. We assume that $\partial \Omega$ is smooth enough and that the surface measure $\int_{\Gamma_{\mathrm{Dir}}} 1 \mathrm{~d} a$ is positive such that Korn's inequality holds; i.e., there exists $c_{\text {Korn }}>0$ such that

$$
\forall u \in \mathcal{F}:\|\mathbf{e}(u)\|_{\mathrm{L}^{2}}^{2} \geq c_{\mathrm{Korn}}\|u\|_{\mathrm{H}^{1}}^{2} .
$$

For more details on Korn's inequality and its consequences, we refer the reader to the [KoO88] or [DuL76].

The stored-energy potential takes the following form:

$$
\begin{aligned}
\mathcal{E}(t, u, z) \stackrel{\text { def }}{=} & \int_{\Omega}\left(W\left(x, \mathbf{e}\left(u+u_{\text {Dir }}(t)\right)(x), z(x), \theta_{\text {appl }}(t, x)\right)+\frac{\sigma}{2}|\nabla z(x)|^{2}\right) \mathrm{d} x \\
& -\langle l(t), u\rangle
\end{aligned}
$$

where the stored-energy density $W: \Omega \times \mathbb{R}_{\text {sym }}^{d \times d} \times Z \times\left[\theta_{\min }, \theta_{\max }\right] \rightarrow \mathbb{R}$ describes the material behavior. Here $\sigma$ is a positive coefficient that is expected to measure some nonlocal interaction effect for the internal variable $z$ and $l(t)$ denotes an applied mechanical loading of the form

$$
\langle l(t), u\rangle \stackrel{\text { def }}{=} \int_{\Omega} f_{\text {appl }}(t, x) \cdot u(x) \mathrm{d} x+\int_{\partial \Omega} g_{\text {appl }}(t, x) \cdot u(x) \mathrm{d} \gamma .
$$

The main point in the model is the choice of the stored-energy density $W$. For notational simplicity, we will omit any dependence on the material point $x \in \Omega$, as it is standard to generalize the approach to this case. For the pure phases $z=\widehat{e}_{k}$ it is clear that $W\left(\cdot, \widehat{e}_{k}, \cdot\right): \mathbb{R}_{\mathrm{sym}}^{d \times d} \times\left[\theta_{\text {min }}, \theta_{\text {max }}\right] \rightarrow \mathbb{R}$ can be adjusted to the measured elasticity constants of this phase. However, the choice for true mixtures $z \in Z$ is not so obvious. In [MiT99, Mie00, MTL02] it was suggested to derive $W$ as a mixture function via cross-quasi convexification:

$$
\begin{array}{r}
W_{\text {mix }}(e, z, \theta)=\inf \left\{\int_{\mathbb{T}_{d}} W(e+\mathbf{e}(\widetilde{v})(y), \widetilde{z}(y), \theta) \mathrm{d} y \mid \widetilde{v} \in \mathrm{H}^{1}\left(\mathbb{T}_{d} ; \mathbb{R}^{d}\right),\right. \\
\left.z=\int_{\mathbb{T}_{d}} \widetilde{z}(y) \mathrm{d} y, \widetilde{z} \in \mathrm{L}^{1}\left(\mathbb{T}_{d} ;\left\{\widehat{e}_{1}, \ldots, \widehat{e}_{N}\right\}\right)\right\},
\end{array}
$$

where $\mathbb{T}_{d}=(\mathbb{R} / \mathbb{Z})^{d}$ is the $d$-dimensional torus; i.e., $\widetilde{v}$ and $\widetilde{z}$ are periodic functions.

In [HaG02, GMH02, GHH07] this function is called free energy of mixing. The point of this construction is that $W(\cdot, z, \theta)$ is still quasi-convex, which is an essential prerequisite for constructing solutions. All this theory was developed for fixed temperature levels and may be much too difficult to be carried through for given material models for the pure phases. In [Mie07, eq. (3.7)] another modeling idea is used by interpolating in a thermal way, such that for convex $W\left(\cdot, \widehat{e}_{k}, \theta\right), k=1, \ldots, N$, the resulting function $W(\cdot, z, \theta)$ is still convex. 
If each $W\left(\cdot, \widehat{e}_{k}, \theta\right)$ is a quadratic function and the associated elasticity tensor is the same for all phases, then it is shown in [Mie00, MTL02] that $W$ takes the form

$$
\begin{aligned}
W_{\text {mix }}(e, z, \theta) & =\sum_{k=1}^{N} z_{k}\left[\frac{1}{2}\left(e-E_{k}(\theta)\right): \mathbb{C}(\theta):\left(e-E_{k}(\theta)\right)+w_{k}^{\text {therm }}(\theta)\right]+w_{\operatorname{mix}}(z, \theta) \\
& =\frac{1}{2}(e-E(z, \theta)): \mathbb{C}(\theta):(e-E(z, \theta))+\widetilde{w}(z, \theta)
\end{aligned}
$$

where $\mathbb{C}(\theta)$ denotes the elasticity tensor, $E_{k}(\theta)$ is the transformation strain of phase $k$ with $E(z, \theta) \stackrel{\text { def }}{=} \sum_{k=1}^{N} z_{k} E_{k}(\theta)$ being the effective transformation strain for a mixture, and $w_{\text {mix }}(\cdot, \theta): Z \rightarrow(-\infty, 0]$ is convex and satisfies $w_{\text {mix }}\left(\widehat{e}_{k}, \theta\right)=0$ for all $k=$ $1, \ldots, N$. In [CaP01, HaG02, GMH02, GHH07] it was shown that this model can be used quite effectively in engineering applications. See section 6 for more discussion of the mixture function $W$.

Our functional $\mathcal{E}$ also includes a gradient term $\frac{\sigma}{2}|\nabla z|^{2}$ which is mainly introduced for mathematical purposes. It will be essential to introduce this term for obtaining the necessary compactness of the abstract theory. After we have averaged the microstructure by allowing for nontrivial phase mixtures, we have to penalize to drastic changes in the mixture composition. This has the disadvantage that we cannot allow for interfaces between the pure austenite and a twined pair of martensite variants (also called habit plane). However, our theory would work equally well if the gradient term would be replaced by a weaker term like

$$
\int_{\Omega \times \Omega} \sigma \frac{|z(y)-z(x)|^{2}}{|y-x|^{d+2 s}} \mathrm{~d} x \mathrm{~d} y
$$

for $s \in(0,1)$, which leads to the Sobolev space $\mathrm{H}^{s}(\Omega)$ instead of $\mathrm{H}^{1}(\Omega)$ for the definition of $\mathcal{Z}$. For $s<1 / 2$ piecewise constant functions are contained in $\mathrm{H}^{s}(\Omega)$, and hence habit planes would have finite energy. For notational convenience we restrict ourselves to the case $s=1$.

To model the hysteretic behavior of shape-memory materials, we also have to describe the dissipation as a constitutive law, since this is largely independent of the energy landscape; cf. [Rou02, AGR03, Rou04]. Again, the energy dissipated in a phase transformation between two pure phases can be measured given the values $D\left(x, \widehat{e}_{j}, \widehat{e}_{k}\right)$. It is shown in [MTL02] that from these values there is a canonical way (via optimal transport theory) to find a function $D: \Omega \times Z \times Z \rightarrow[0, \infty)$ such that the dissipation between two states $z_{0}, z_{1} \in Z$ takes the form

$$
D\left(x, z_{0}, z_{1}\right)=\psi\left(x, z_{1}-z_{0}\right),
$$

where the dissipation potential $\psi(x, \cdot): \mathbb{R}_{0}^{N} \rightarrow \mathbb{R}$, with $\mathbb{R}_{0}^{N} \stackrel{\text { def }}{=}\left\{v \in \mathbb{R}^{N} \mid \sum_{j=1}^{N} v_{j}=\right.$ $0\}$, is convex and positively homogeneous of degree 1 ; i.e., for all $\gamma \geq 0$ and $v \in \mathbb{R}_{0}^{N}$, $\psi(x, \gamma v)=\gamma \psi(x, v)$.

At the moment, we do not assume that $D$ is defined via $\psi$ but postulate a dissipation distance $D: Z \times Z \rightarrow[0, \infty)$ satisfying the following two properties which imply that $D$ is a quasi distance (as for $W$, we suppress the $x$-dependence of $D$ from now on):

$$
\begin{aligned}
& D\left(z_{0}, z_{1}\right)=0 \quad \Longleftrightarrow \quad z_{0}=z_{1}, \\
& \forall z_{1}, z_{2}, z_{3} \in Z: \quad D\left(z_{1}, z_{3}\right) \leq D\left(z_{1}, z_{2}\right)+D\left(z_{2}, z_{3}\right) .
\end{aligned}
$$


Note that symmetry $D\left(z_{1}, z_{2}\right)=D\left(z_{2}, z_{1}\right)$ is not needed, which may be useful, as the dissipated energy for transforming from austenite to martensite and vice versa may be different. Finally, the total dissipation distance between two internal states $z_{0}, z_{1} \in \mathcal{Z}$ is defined via

$$
\mathcal{D}\left(z_{0}, z_{1}\right) \stackrel{\text { def }}{=} \int_{\Omega} D\left(z_{0}(x), z_{1}(x)\right) \mathrm{d} x
$$

The evolution is assumed to be governed by the energetic formulation of rateindependent processes as introduced in [MTL02, MiT04, Mie05, MaM05, FrM06]. More precisely, a function $q:[0, T] \rightarrow \mathcal{Q}$ is called an energetic solution of the rateindependent problem associated with $\mathcal{E}$ and $\mathcal{D}$ if for all $t \in[0, T]$ the global stability condition (S) and the global energy balance (E) are satisfied, i.e.,

$$
\begin{aligned}
& \text { (S) } \forall \bar{q}=(\bar{u}, \bar{z}) \in \mathcal{Q}: \mathcal{E}(t, q(t)) \leq \mathcal{E}(t, \bar{q})+\mathcal{D}(z(t), \bar{z}) \\
& \text { (E) } \mathcal{E}(t, q(t))+\operatorname{Var}_{\mathcal{D}}(z ;[0, t])=\mathcal{E}(0, q(0))+\int_{0}^{t} \partial_{s} \mathcal{E}(s, q(s)) \mathrm{d} s .
\end{aligned}
$$

The dissipation $\operatorname{Var}_{\mathcal{D}}$ is defined via

$$
\operatorname{Var}_{\mathcal{D}}(z ;[r, s]) \stackrel{\text { def }}{=} \sup \left\{\sum_{j=1}^{n} \mathcal{D}\left(z\left(t_{j-1}\right), z\left(t_{j}\right)\right) \mid n \in \mathbb{N}, r \leq t_{0}<t_{1}<\cdots<t_{n} \leq s\right\}
$$

for all $(r, s) \in[0, T]^{2}$ such that $r<s$.

As detailed in [MiT04, Mie05], we can interpret the energetic formulation as a weak form of the associated evolution law defined by elastic equilibrium and the flow rule for the internal variable $z$. In particular, if the functional $\mathcal{E}(t, \cdot)$ is convex and $D$ is given in the form (2.6), then the energetic formulation $(\mathrm{S})$ and $(\mathrm{E})$ is equivalent to the following doubly nonlinear evolution law:

$$
\text { elastic equilibrium }\left\{\begin{array}{l}
-\operatorname{div}_{e} W\left(\mathbf{e}\left(u+u_{\text {Dir }}\right), z, \theta_{\text {appl }}\right)=f_{\text {appl }} \text { in } \Omega \\
u=u_{\text {Dir }} \text { on } \Gamma_{\text {Dir }}, \\
\partial_{e} W\left(\mathbf{e}\left(u+u_{\text {Dir }}\right), z, \theta_{\text {appl }}\right) \nu=g_{\text {appl }} \text { on } \Gamma_{\text {Neu }}
\end{array}\right.
$$

flow rule

$$
0 \in \partial \psi(\dot{z})+\partial_{z} W\left(\mathbf{e}\left(u+u_{\text {Dir }}\right), z, \theta_{\text {appl }}\right)+\partial \chi_{Z}(z) \text { in } \Omega
$$

where $\partial$ without a subscript denotes the set-valued subdifferential of a convex function. In fact, under the assumptions of section 4 the energetic solutions satisfy (2.9) as well.

3. The existence result. In this section we collect the assumptions on the constitutive functions $W$ and $D$ and on the data $\theta_{\text {appl }}, l$, and $u_{\text {Dir }}$ that allow us to apply the abstract existence theory for energetic solutions to the rate-independent system $(\mathcal{Q}, \mathcal{E}, \mathcal{D})$. Thus, we will just check that the assumptions of the result in [FrM06] are satisfied. In fact, virtually the same assumptions will be used in section 5 to obtain the convergence result, which is again an existence result, as existence is not assumed beforehand. After stating the existence result, we will collect a number of properties of the energy functional $\mathcal{E}$, which will be useful in the later sections. 
For the prescribed temperature profile $\theta_{\text {appl }}$, the external loading $l$, and the Dirichlet boundary condition $u_{\text {Dir }}$ we assume

$$
\begin{aligned}
\theta_{\text {appl }} & \in \mathrm{C}^{1}\left([0, T] ; \mathrm{L}^{\infty}\left(\Omega ;\left[\theta_{\min }, \theta_{\max }\right]\right)\right), \\
l & \in \mathrm{C}^{1}\left([0, T] ;\left(\mathrm{H}^{1}\left(\Omega ; \mathbb{R}^{d}\right)\right)^{\prime}\right), \\
u_{\text {Dir }} & \in \mathrm{C}^{1}\left([0, T] ; \mathrm{H}^{1}\left(\Omega ; \mathbb{R}^{d}\right)\right) .
\end{aligned}
$$

For the stored-energy density $W: \mathbb{R}_{\text {sym }}^{d \times d} \times Z \times\left[\theta_{\min }, \theta_{\max }\right] \rightarrow \mathbb{R}$ we impose the following conditions. In section 6 , we will show that these conditions are satisfied by some of the functions $W$ introduced in the previous section.

Assumptions on $\boldsymbol{W}$. There exist positive constants $C, c, C_{0}^{W}, C_{1}^{W}, C^{\theta}, C_{0}^{\theta}$, $C_{1}^{\theta}, C^{e}, C_{0}^{e}, C_{1}^{e}$, an exponent $\widehat{p} \in(0,2)$, and a nondecreasing function $\omega:[0, \infty) \rightarrow$ $[0, \infty)$ with $\lim _{\tau \rightarrow 0^{+}} \omega(\tau)=0$ such that for all $e, e_{1}, e_{2} \in \mathbb{R}_{\mathrm{sym}}^{d \times d}, z, z_{1}, z_{2} \in Z$, and $\theta, \theta_{1}, \theta_{2} \in\left[\theta_{\min }, \theta_{\max }\right]$ we have

$$
\begin{aligned}
& W(\cdot, z, \theta) \text { is strictly convex, } \\
& W, \partial_{\theta} W \in \mathrm{C}^{0}\left(\mathbb{R}_{\mathrm{sym}}^{d \times d} \times Z \times\left[\theta_{\min }, \theta_{\max }\right] ; \mathbb{R}\right), \\
& \partial_{e} W \in \mathrm{C}^{0}\left(\mathbb{R}_{\mathrm{sym}}^{d \times d} \times Z \times\left[\theta_{\min }, \theta_{\max }\right] ; \mathbb{R}_{\mathrm{sym}}^{d \times d}\right), \\
& c\left(|e|^{2}+|z|^{2}\right)-C \leq W(e, z, \theta) \leq C\left(|e|^{2}+|z|^{2}\right)+C, \\
& \left|\partial_{e} W(e, z, \theta)\right|^{2}+\left|\partial_{\theta} W(e, z, \theta)\right| \leq C_{1}^{W}\left(W(e, z, \theta)+C_{0}^{W}\right), \\
& \left|\partial_{\theta} W\left(e, z, \theta_{1}\right)-\partial_{\theta} W\left(e, z, \theta_{2}\right)\right| \leq C_{1}^{\theta}\left(W\left(e, z, \theta_{1}\right)+C_{0}^{\theta}\right) \omega\left(\left|\theta_{1}-\theta_{2}\right|\right), \\
& \left|\partial_{e} W\left(e, z, \theta_{1}\right)-\partial_{e} W\left(e, z, \theta_{2}\right)\right|^{2} \leq C_{1}^{e}\left(W\left(e, z, \theta_{1}\right)+C_{0}^{e}\right) \omega\left(\left|\theta_{1}-\theta_{2}\right|\right), \\
& \left|\partial_{\theta} W\left(e_{1}, z_{1}, \theta\right)-\partial_{\theta} W\left(e_{2}, z_{2}, \theta\right)\right| \\
& \leq C^{\theta}\left(\left|e_{1}-e_{2}\right|+\left|z_{1}-z_{2}\right|\right)\left(1+\left|e_{1}+e_{2}\right|+\left|z_{1}+z_{2}\right|\right), \\
& \left|\partial_{e} W\left(e_{1}, z_{1}, \theta\right)-\partial_{e} W\left(e_{2}, z_{2}, \theta\right)\right| \leq C^{e}\left(\left|e_{1}-e_{2}\right|+\left|z_{1}-z_{2}\right|\right), \\
& \left|W\left(e, z_{1}, \theta\right)-W\left(e, z_{2}, \theta\right)\right| \leq C(1+|e|)^{\widehat{p}} \omega\left(\left|z_{1}-z_{2}\right|\right) .
\end{aligned}
$$

For the dissipation distance we impose (2.7) and

$$
\exists c_{1}, c_{2}>0 \forall z_{1}, z_{2} \in Z: \quad c_{1}\left|z_{1}-z_{2}\right| \leq D\left(z_{1}, z_{2}\right) \leq c_{2}\left|z_{1}-z_{2}\right| .
$$

We prove now that the energetic formulation $(\mathrm{S})$ and $(\mathrm{E})$ has at least one solution $q:[0, T] \rightarrow \mathcal{Q}$ for any given stable initial data $q_{0}=\left(u_{0}, z_{0}\right) \in \mathcal{Q}$; i.e., $q_{0} \in \mathcal{Q}$ satisfies the global stability condition $(\mathrm{S})$ at $t=0$. The existence theory for $(\mathrm{S})$ and $(\mathrm{E})$ has been developed in [MaM05, FrM06, Mie05] and is based on the construction of a sequence of incremental minimization problems. More precisely, for a given partition $\Pi=\left\{0=t<t_{1}<\cdots<t_{n}=T\right\}$, we define the incremental problems as follows:

$$
(\mathrm{IP})_{\Pi}\left\{\begin{array}{l}
\text { for } k=1, \ldots, n \text { find } \\
q_{k} \stackrel{\text { def }}{=}\left(u_{k}, z_{k}\right) \in \operatorname{Argmin}\left\{\mathcal{E}\left(t_{k}, \bar{q}\right)+\mathcal{D}\left(z_{k-1}, \bar{z}\right) \mid \bar{q}=(\bar{u}, \bar{z}) \in \mathcal{Q}\right\} .
\end{array}\right.
$$


Let the piecewise constant interpolant $q^{\Pi}:[0, T] \rightarrow \mathcal{Q}$ be defined by $q^{\Pi}(t)=q_{j}$ for $t \in\left[t_{j}, t_{j+1}\right)$ for $j=0, \ldots, n-1$ and $q^{\Pi}(T)=q_{n}$. Then one shows that a subsequence of $\left(q^{\Pi}\right)_{\Pi}$ has a limit, and this limit function satisfies the energetic formulation (S) and $(\mathrm{E})$.

Note that our statement given here is slightly stronger than the one obtained in the abstract setting. First, we state that not only the $z$-component of $q$ converges but also the $u$-component. Second, we provide strong convergence in $\mathcal{Q}$, i.e., in the norm topology of $\mathrm{H}^{1}(\Omega)$.

THEOREM 3.1. Assume that $W$ and $D$ satisfy (2.7), (3.2), and (3.3) and that the data $u_{\text {Dir }}, l$, and $\theta_{\text {appl }}$ satisfy (3.1). Let $q_{0} \in \mathcal{Q}$ be stable for $t=0$. Then there exists an energetic solution $q=(u, z):[0, T] \rightarrow \mathcal{Q}$ such that $q_{0}=(u(0), z(0))$ and

$$
\begin{aligned}
& u \in \mathrm{L}^{\infty}\left([0, T] ; \mathrm{H}^{1}\left(\Omega ; \mathbb{R}^{d}\right)\right), \\
& z \in \mathrm{L}^{\infty}\left([0, T] ; \mathrm{H}^{1}(\Omega ; Z)\right) \cap \operatorname{BV}\left([0, T] ; \mathrm{L}^{1}(\Omega ; Z)\right) .
\end{aligned}
$$

Moreover, let $\Pi_{k}=\left\{0=t_{0}^{k}<t_{1}^{k}<\cdots<t_{N_{k}}^{k}=T\right\}, k \in \mathbb{N}$, be a sequence of partitions with fineness $\Delta\left(\Pi_{k}\right) \stackrel{\text { def }}{=} \max \left\{t_{j}^{k}-t_{j-1}^{k}: j=1, \ldots, N_{k}\right\}$ tending to 0 for $k \rightarrow \infty$. Let $q^{\Pi_{k}} \stackrel{\text { def }}{=}\left(u^{\Pi_{k}}, z^{\Pi_{k}}\right):[0, T] \rightarrow \mathcal{Q}$ be the piecewise constant interpolants associated with the incremental problems $(\mathrm{IP})_{\Pi_{k}}$; then there exist a subsequence $\bar{q}_{n} \stackrel{\text { def }}{=} q^{\Pi_{k_{n}}}$ and an energetic solution $\widehat{q}:[0, T] \rightarrow \mathcal{Q}$ such that for all $t \in[0, T]$ the following holds:

$$
\begin{aligned}
\bar{q}_{n}(t) & \rightarrow \widehat{q}(t) \text { in } \mathcal{Q}, \\
\mathcal{E}\left(t, \bar{q}_{n}(t)\right) & \rightarrow \mathcal{E}(t, \widehat{q}(t)), \\
\operatorname{Var}_{\mathcal{D}}\left(\bar{z}_{n} ;[0, t]\right) & \rightarrow \operatorname{Var}_{\mathcal{D}}(\widehat{z} ;[0, t]) .
\end{aligned}
$$

Proof. We use the abstract result of [FrM06], which relies on the following abstract assumptions (i)-(v), where $\mathcal{F}$ and $\mathcal{Z}$ are considered as topological spaces carrying the weak topology of $\mathrm{H}^{1}(\Omega)$ :

(i) $\forall z_{1}, z_{2}, z_{3} \in \mathcal{Z}: \mathcal{D}\left(z_{1}, z_{2}\right)=0 \Longleftrightarrow z_{1}=z_{2}$ and $\mathcal{D}\left(z_{1}, z_{3}\right) \leq \mathcal{D}\left(z_{1}, z_{2}\right)+$ $\mathcal{D}\left(z_{2}, z_{3}\right)$

(ii) $\mathcal{D}: \mathcal{Z} \times \mathcal{Z} \rightarrow[0, \infty)$ is continuous;

(iii) $\forall t \in[0, T]: \mathcal{E}(t, \cdot): \mathcal{Q} \rightarrow[0, \infty)$ has compact sublevels;

(iv) there exists $C_{0}^{\mathcal{E}}, C_{1}^{\mathcal{E}}>0$ such that $\forall q \in \mathcal{Q}$

$$
\mathcal{E}(t, q)<\infty \quad \Longrightarrow \quad\left\{\begin{array}{l}
\mathcal{E}(\cdot, q) \in \mathrm{C}^{1}([0, T]) \text { and } \\
\left|\partial_{t} \mathcal{E}(t, q)\right| \leq C_{1}^{\mathcal{E}}\left(\mathcal{E}(t, q)+C_{0}^{\mathcal{E}}\right)
\end{array}\right.
$$

(v) $\forall \eta>0 \forall \varepsilon>0 \exists \delta>0 \forall q \in \mathcal{Q} \forall t_{1}, t_{2} \in[0, T]$ :

$$
\left(\mathcal{E}(0, q) \leq \eta,\left|t_{1}-t_{2}\right| \leq \delta\right) \quad \Longrightarrow \quad\left|\partial_{t} \mathcal{E}\left(t_{1}, q\right)-\partial_{t} \mathcal{E}\left(t_{2}, q\right)\right|<\varepsilon .
$$

Property (i) follows from the definition (2.8) of the dissipation potential $\mathcal{D}$ and the conditions (2.7) and (3.3). The latter condition also implies that $\mathcal{D}\left(z_{1}, z_{2}\right)$ is bounded from above and below by the norm of $z_{1}-z_{2}$ in $\mathrm{L}^{1}(\Omega)$. Hence, $\mathcal{D}$ is strongly continuous in $\mathrm{L}^{1}(\Omega)$, and the compact embedding of $\mathrm{H}^{1}(\Omega)$ into $\mathrm{L}^{1}(\Omega)$ provides (ii).

On the one hand, $\mathcal{E}(t, \cdot)$ is coercive because of $(3.2 \mathrm{~d})$ and $(2.2)$. Moreover, $\mathcal{E}(t, \cdot)$ is weakly lower semicontinuous, as the integrand is convex in $(\nabla u, \nabla z)$ and continuous in $(u, z, \nabla u, \nabla z)$. This provides (iii). Finally, (iv) and (v) will be obtained in Proposition 3.3 . 
Since the assumptions (i)-(v) are fulfilled, [FrM06, Thm. 3.4] or [Mie05, Thm. 5.2] are applicable, and the statement of the theorem follows, except for $(3.4 \mathrm{a})$, where only $\bar{z}_{n}(t) \rightarrow \widehat{z}(t)$ is inferred.

To obtain the convergence of $\bar{u}_{n}(t)$ we note that by construction $\bar{u}_{n}(t)$ minimizes the energy $\mathcal{E}\left(\tau(n, t), \cdot, \bar{z}_{n}(t)\right)$, where $\tau(n, t)$ is the largest point in $\Pi_{k_{n}}$ not exceeding $t$. Since we have $\tau(n, t) \rightarrow t$ and $\bar{z}_{n}(t) \rightarrow \widehat{z}(t)$, we may infer part of Lemma 3.4 to obtain (3.4a).

Now we collect some properties of $\mathcal{E}$ and $\mathcal{D}$ that we will use in the next sections.

LEMmA 3.2. Let the assumptions (3.1), (3.2a), (3.2b), (3.2d), (2.7), and (3.3) hold. Then, the energy functional $\mathcal{E}:[0, T] \times \mathcal{Q} \rightarrow \mathbb{R}$ is weakly lower semicontinuous and strongly continuous, and is coercive:

$$
\exists C_{0}, c_{0}>0 \forall(t, q) \in[0, T] \times \mathcal{Q}: C_{0}\|q\|_{\mathcal{Q}}^{2}-c_{0} \leq \mathcal{E}(t, q) \leq c_{0}\|q\|_{\mathcal{Q}}^{2}+c_{0} .
$$

The dissipation distance $\mathcal{D}: \mathcal{Z} \times \mathcal{Z} \rightarrow[0, \infty)$ is weakly continuous.

Proof. First, let us observe that Korn's inequality (2.2), Young's inequality, and (3.2d) lead to

$\mathcal{E}(t, q) \geq \frac{c c_{\mathrm{Korn}}}{4}\|u\|_{\mathrm{H}^{1}}^{2}+\min \left(c, \frac{\sigma}{2}\right)\|z\|_{\mathrm{H}^{1}}^{2}-C|\Omega|-\frac{1}{c c_{\mathrm{Korn}}}\|l(t)\|_{\left(\mathrm{H}^{1}\right)^{\prime}}^{2}-c\left\|\mathbf{e}\left(u_{\mathrm{Dir}}(t)\right)\right\|_{\mathrm{L}^{2}}^{2}$

for all $(t, q) \in[0, T] \times \mathcal{Q}$. Similarly, $(3.2 \mathrm{~d})$ implies

$\mathcal{E}(t, q) \leq\left(2 C+\frac{1}{2}\right)\|u\|_{\mathrm{H}^{1}}^{2}+\max \left(C, \frac{\sigma}{2}\right)\|z\|_{\mathrm{H}^{1}}^{2}+C|\Omega|+\frac{1}{2}\|l(t)\|_{\left(\mathrm{H}^{1}\right)^{\prime}}^{2}+2 C\left\|\mathbf{e}\left(u_{\mathrm{Dir}}(t)\right)\right\|_{\mathrm{L}^{2}}^{2}$,

and, by using (3.1), we may conclude that (3.5) holds.

The weak lower semicontinuity of $\mathcal{E}(t, \cdot): \mathcal{Q} \rightarrow \mathbb{R}$ follows from the convexity of the integrand in highest derivatives of $(u, z)$, namely $(\mathbf{e}(u), \nabla z)$. Weak continuity of $\mathcal{D}$ is a consequence of the strong continuity of $\mathcal{D}$ with respect to the norm in $\mathrm{L}^{1}(\Omega)$ and the compact embedding of $\mathcal{Z} \subset \mathrm{H}^{1}\left(\Omega ; \mathbb{R}^{N}\right)$ into $\mathrm{L}^{1}\left(\Omega ; \mathbb{R}^{N}\right)$.

It remains to show the strong continuity of $\mathcal{E}$. For this assume $\left(t_{n}, q_{n}\right) \rightarrow\left(t_{*}, q_{*}\right)$. Using (3.5) the sequence $\left(\mathcal{E}\left(t_{n}, q_{n}\right)\right)_{n \in \mathbb{N}}$ is bounded, and we may choose a subsequence $\left(t_{n_{j}}, q_{n_{j}}\right)_{j \in \mathbb{N}}$ such that

$$
\begin{aligned}
& \mathcal{E}\left(t_{n_{j}}, q_{n_{j}}\right) \rightarrow \mathcal{E}_{*}, \\
& \forall \text { a.a. } x \in \Omega:\left(e_{n_{j}}(x), z_{n_{j}}(x), \theta_{\text {appl }}\left(t_{n_{j}}, x\right)\right) \rightarrow\left(e_{*}(x), z_{*}(x), \theta_{\text {appl }}(t, x)\right), \\
& \exists \gamma \in \mathrm{L}^{2}(\Omega) \forall j \in \mathbb{N}:\left|\left(e_{n_{j}}, z_{n_{j}}\right)\right| \leq \gamma \text { a.e. in } \Omega
\end{aligned}
$$

with $e_{n_{j}}=\mathbf{e}\left(u_{n_{j}}+u_{\text {Dir }}\left(t_{n_{j}}\right)\right)$ for all $j \in \mathbb{N}$. Thus, we may pass to the limit in $\mathcal{E}\left(t_{n_{j}}, q_{n_{j}}\right)=\int_{\Omega} W\left(\mathbf{e}\left(u_{n_{j}}+u_{\mathrm{Dir}}\left(t_{n_{j}}\right)\right), z_{n_{j}}, \theta_{\mathrm{appl}}\left(t_{n_{j}}\right)\right) \mathrm{d} x+\frac{\sigma}{2}\left\|\nabla z_{n_{j}}\right\|_{\mathrm{L}^{2}}^{2}-\left\langle l\left(t_{n_{j}}\right), u_{n_{j}}\right\rangle$,

by applying Lebesgue's theorem and using (3.1). We obtain $\mathcal{E}_{*}=\lim _{j \rightarrow \infty} \mathcal{E}\left(t_{n_{j}}, q_{n_{j}}\right)=$ $\mathcal{E}\left(t_{*}, q_{*}\right)$ and, by uniqueness of the limit, the whole sequence $\left(\mathcal{E}\left(t_{n}, q_{n}\right)\right)_{n \in \mathbb{N}}$ converges to $\mathcal{E}\left(t_{*}, q_{*}\right)$.

We now check the last two assumptions (iv) and (v) of the abstract result of [FrM06], which are needed to obtain Theorem 3.1. To do so, we first observe that the regularity assumptions on $W$ and the data $u_{\text {Dir }}, l$, and $\theta_{\text {appl }}$ imply that $\mathcal{E}(\cdot, q) \in$ 
$C^{1}([0, T])$ for all $q \in \mathcal{Q}$ and we derive an explicit formula for $\partial_{t} \mathcal{E}(\cdot, q)$. Then using the assumptions $(3.2 \mathrm{e})$ to $(3.2 \mathrm{i})$ we obtain an estimate of $\left|\partial_{t} \mathcal{E}(t, q)\right|$ in terms of $\mathcal{E}(t, q)$ and establish $(\mathrm{v})$, which can be interpreted as a uniform continuity property for $\partial_{t} \mathcal{E}(\cdot, q)$ on energy sublevels.

Proposition 3.3. Let us assume that (3.1) and (3.2b) to (3.2i) hold. Then $\mathcal{E}$ satisfies the following properties:

(P1) Let $q=(u, z) \in \mathcal{Q}$. Then $\mathcal{E}(\cdot, q)$ lies in $\mathrm{C}^{1}([0, T])$ and

$$
\begin{aligned}
\partial_{t} \mathcal{E}(t, q)= & \int_{\Omega} \partial_{e} W\left(\mathbf{e}\left(u+u_{\text {Dir }}(t)\right), z, \theta_{\text {appl }}(t)\right) \mathbf{e}\left(\dot{u}_{\text {Dir }}(t)\right) \mathrm{d} x \\
& +\int_{\Omega} \partial_{\theta} W\left(\mathbf{e}\left(u+u_{\operatorname{Dir}}(t)\right), z, \theta_{\text {appl }}(t)\right) \dot{\theta}_{\text {appl }}(t) \mathrm{d} x-\langle i(t), u\rangle .
\end{aligned}
$$

(P2) There exist $C_{0}^{\mathcal{E}}, C_{1}^{\mathcal{E}}>0$ such that $\left|\partial_{t} \mathcal{E}(t, q)\right| \leq C_{1}^{\mathcal{E}}\left(\mathcal{E}(t, q)+C_{0}^{\mathcal{E}}\right)$ for all $(t, q) \in$ $[0, T] \times \mathcal{Q}$.

(P3) For each $\varepsilon>0$ and $E \in \mathbb{R}^{+}$there exists $\delta>0$ such that for all $(s, t, q) \in$ $[0, T]^{2} \times \mathcal{Q}$ with $\mathcal{E}(0, q) \leq E$ and $|s-t| \leq \delta$ we have $\left|\partial_{t} \mathcal{E}(s, q)-\partial_{t} \mathcal{E}(t, q)\right|<\varepsilon$.

Estimate (P2) together with Gronwall's lemma leads to

$$
\forall s, t \in[0, T]: \mathcal{E}(t, q) \leq \exp \left(C_{1}^{\mathcal{E}}|t-s|\right)\left(\mathcal{E}(s, q)+C_{0}^{\mathcal{E}}\right)-C_{0}^{\mathcal{E}} .
$$

This estimate is crucial to derive a priori estimates, also in the time-discrete setting.

Proof. First, we infer from $(3.1)$ and $(3.2 \mathrm{~b})$ to $(3.2 \mathrm{e})$ that $\mathcal{E}(\cdot, q) \in \mathrm{C}^{1}([0, T])$ and that (3.6) holds.

For (P2), one can see that assumptions (3.1) and Cauchy-Schwarz's inequality lead to

$$
\begin{aligned}
\left|\partial_{t} \mathcal{E}(t, q)\right| \leq & \frac{1}{2} \int_{\Omega}\left|\partial_{e} \widetilde{W}\left(t, \theta_{\mathrm{appl}}(t)\right)\right|^{2} \mathrm{~d} x+\frac{1}{2}\left\|\mathbf{e}\left(\dot{u}_{\mathrm{Dir}}(t)\right)\right\|_{\mathrm{L}^{2}}^{2} \\
& +\Theta \int_{\Omega}\left|\partial_{\theta} \widetilde{W}\left(t, \theta_{\mathrm{appl}}(t)\right)\right| \mathrm{d} x+\frac{1}{2}\|u\|_{\mathrm{H}^{1}}^{2}+\frac{1}{2}\|\dot{l}(t)\|_{\left(\mathrm{H}^{1}\right)^{\prime}}^{2}
\end{aligned}
$$

where $\Theta \stackrel{\text { def }}{=}\left\|\dot{\theta}_{\text {appl }}(\cdot)\right\|_{\mathrm{C}^{0}\left([0, T] ; \mathrm{L}^{\infty}\right)}$ and $\widetilde{W}(s, \theta) \stackrel{\text { def }}{=} W\left(\mathbf{e}\left(u+u_{\operatorname{Dir}}(s)\right), z, \theta\right)$. Carrying (3.2e) into (3.8), we have

$$
\begin{aligned}
\left|\partial_{t} \mathcal{E}(t, q)\right| \leq & \left(\frac{1}{2}+\Theta\right) \int_{\Omega} C_{1}^{W}\left(\widetilde{W}\left(t, \theta_{\text {appl }}(t)\right)+C_{0}^{W}\right) \mathrm{d} x \\
& +\frac{1}{2}\|u\|_{\mathrm{H}^{1}}^{2}+\frac{1}{2}\left\|\mathbf{e}\left(\dot{u}_{\mathrm{Dir}}(t)\right)\right\|_{\mathrm{L}^{2}}^{2}+\frac{1}{2}\|\dot{l}(t)\|_{\left(\mathrm{H}^{1}\right)^{\prime}}^{2},
\end{aligned}
$$

which implies using (2.3) that

$$
\left|\partial_{t} \mathcal{E}(t, q)\right| \leq\left(\frac{1}{2}+C_{1}^{W}\left(\frac{1}{2}+\Theta\right)\right)\left(\mathcal{E}(t, q)+\|u\|_{\mathrm{H}^{1}}^{2}+C_{1}\right)
$$

where $C_{1} \stackrel{\text { def }}{=}\left\|\mathbf{e}\left(\dot{u}_{\mathrm{Dir}}\right)\right\|_{\mathrm{C}^{0}\left([0, T] ; \mathrm{L}^{2}\right)}^{2}+\|l\|_{\mathrm{C}^{0}\left([0, T] ;\left(\mathrm{H}^{1}\right)^{\prime}\right)}^{2}+\| \dot{l}_{\mathrm{C}^{0}\left([0, T] ;\left(\mathrm{H}^{1}\right)^{\prime}\right)}^{2}+C_{0}^{W}|\Omega|$. Using (3.5) in (3.9), the announced result (P2) follows immediately. 
To derive estimate (P3) we use the decompositions

$$
\left|\partial_{t} \mathcal{E}(s, q)-\partial_{t} \mathcal{E}(t, q)\right| \leq \mathcal{I}_{e}(s, t)+\mathcal{I}_{\theta}(s, t)+\mathcal{I}_{l}(s, t)
$$

where

$\mathcal{I}_{e}(s, t) \stackrel{\text { def }}{=} \int_{\Omega}\left|\partial_{e} \widetilde{W}\left(s, \theta_{\text {appl }}(s)\right) \mathbf{e}\left(\dot{u}_{\text {Dir }}(s)\right)-\partial_{e} \widetilde{W}\left(t, \theta_{\text {appl }}(t)\right) \mathbf{e}\left(\dot{u}_{\text {Dir }}(t)\right)\right| \mathrm{d} x$

(3.10c) $\mathcal{I}_{\theta}(s, t) \stackrel{\text { def }}{=} \int_{\Omega}\left|\partial_{\theta} \widetilde{W}\left(s, \theta_{\text {appl }}(s)\right) \dot{\theta}_{\text {appl }}(s)-\partial_{\theta} \widetilde{W}\left(t, \theta_{\text {appl }}(t)\right) \dot{\theta}_{\text {appl }}(t)\right| \mathrm{d} x$,

(3.10d) $\mathcal{I}_{l}(s, t) \stackrel{\text { def }}{=}|\langle\dot{l}(s)-\dot{l}(t), u\rangle| \leq\|\dot{l}(s)-\dot{l}(t)\|_{\left(\mathrm{H}^{1}\right)^{\prime}}\|u\|_{\mathrm{H}^{1}} \leq\|\dot{l}(s)-\dot{l}(t)\|_{\left(\mathrm{H}^{1}\right)^{\prime}} \sqrt{\frac{E+c_{0}}{C_{0}}}$,

where we used (3.5) for the last estimate.

Each term on the right-hand side of $(3.10 \mathrm{a})$ is estimated separately by using the assumptions on $W$ introduced above. Since $\mathcal{E}(0, q) \leq E$, one deduces from $(2.3),(3.5)$, and (3.7) that

$$
\int_{\Omega} \widetilde{W}\left(s, \theta_{\operatorname{appl}}(s)\right) \mathrm{d} x \leq \rho(E)
$$

where $\rho(E) \stackrel{\text { def }}{=} \exp \left(C_{1}^{\mathcal{E}} T\right)\left(E+C_{0}^{\mathcal{E}}\right)-C_{0}^{\mathcal{E}}+\sup _{s \in[0, T]}\|l(s)\|_{\left(\mathrm{H}^{1}\right)^{\prime}} \sqrt{\frac{E+c_{0}}{C_{0}}}$. Using (3.1) the right-hand side of (3.11) is bounded independently of $s$ and $q$. Let us now observe that

$$
\mathcal{I}_{e}(s, t) \leq \int_{\Omega}\left|\partial_{e} \widetilde{W}\left(s, \theta_{\mathrm{appl}}(s)\right)\left(\mathbf{e}\left(\dot{u}_{\mathrm{Dir}}(s)\right)-\mathbf{e}\left(\dot{u}_{\mathrm{Dir}}(t)\right)\right)\right| \mathrm{d} x+\mathcal{I}_{\mathrm{var}}^{e}(s, t)
$$

where

$$
\begin{aligned}
\mathcal{I}_{\text {var }}^{e}(s, t) \stackrel{\text { def }}{=} & \int_{\Omega}\left|\left(\partial_{e} \widetilde{W}\left(s, \theta_{\text {appl }}(s)\right)-\partial_{e} \widetilde{W}\left(t, \theta_{\text {appl }}(s)\right)\right) \mathbf{e}\left(\dot{u}_{\text {Dir }}(t)\right)\right| \mathrm{d} x \\
& +\int_{\Omega}\left|\left(\partial_{e} \widetilde{W}\left(t, \theta_{\text {appl }}(s)\right)-\partial_{e} \widetilde{W}\left(t, \theta_{\text {appl }}(t)\right)\right) \mathbf{e}\left(\dot{u}_{\operatorname{Dir}}(t)\right)\right| \mathrm{d} x .
\end{aligned}
$$

Using Cauchy-Schwarz's inequality and (3.2e), the first term on the right-hand side of (3.12) is estimated by

$$
\begin{aligned}
& \int_{\Omega}\left|\partial_{e} \widetilde{W}\left(s, \theta_{\text {appl }}(s)\right)\left(\mathbf{e}\left(\dot{u}_{\text {Dir }}(s)\right)-\mathbf{e}\left(\dot{u}_{\text {Dir }}(t)\right)\right)\right| \mathrm{d} x \\
& \leq\left(C_{1}^{W} \int_{\Omega} \widetilde{W}\left(s, \theta_{\text {appl }}(s)\right) \mathrm{d} x+C_{1}^{W} C_{0}^{W}|\Omega|\right)^{1 / 2}\left\|\mathbf{e}\left(\dot{u}_{\text {Dir }}(s)\right)-\mathbf{e}\left(\dot{u}_{\text {Dir }}(t)\right)\right\|_{L^{2}} .
\end{aligned}
$$

Introducing (3.11) in the latter estimate, we deduce that there exists $\widetilde{C}_{1}^{E}>0$ such that

$$
\begin{aligned}
& \int_{\Omega}\left|\partial_{e} \widetilde{W}\left(s, \theta_{\text {appl }}(s)\right)\left(\mathbf{e}\left(\dot{u}_{\text {Dir }}(s)\right)-\mathbf{e}\left(\dot{u}_{\text {Dir }}(t)\right)\right)\right| \mathrm{d} x \\
& \leq \widetilde{C}_{1}^{E}\left\|\mathbf{e}\left(\dot{u}_{\text {Dir }}(s)\right)-\mathbf{e}\left(\dot{u}_{\text {Dir }}(t)\right)\right\|_{\mathrm{L}^{2}} .
\end{aligned}
$$


For $\mathcal{I}_{\text {var }}^{e}$ we use (3.2i) and Cauchy-Schwarz's inequality to find

$$
\begin{aligned}
\mathcal{I}_{\mathrm{var}}^{e}(s, t) \leq & \eta\left(C^{e}\left\|\mathbf{e}\left(u_{\mathrm{Dir}}(s)\right)-\mathbf{e}\left(u_{\mathrm{Dir}}(t)\right)\right\|_{\mathrm{L}^{2}}\right. \\
& \left.+\left\|\partial_{e} \widetilde{W}\left(t, \theta_{\mathrm{appl}}(s)\right)-\partial_{e} \widetilde{W}\left(t, \theta_{\mathrm{appl}}(t)\right)\right\|_{\mathrm{L}^{2}}\right),
\end{aligned}
$$

where $\eta \stackrel{\text { def }}{=}\left\|\mathbf{e}\left(\dot{u}_{\text {Dir }}(\cdot)\right)\right\|_{\mathrm{C}^{0}\left([0, T] ; \mathrm{L}^{2}\right)}$. By $(3.1 \mathrm{a})$ we have

$$
\begin{aligned}
\forall_{\text {a.e. }} x \in \Omega: \omega\left(\left|\theta_{\mathrm{appl}}(s, x)-\theta_{\mathrm{appl}}(t, x)\right|\right) & \leq \bar{\omega}_{s, t} \stackrel{\text { def }}{=} \omega\left(\left\|\theta_{\mathrm{appl}}(s)-\theta_{\mathrm{appl}}(t)\right\|_{\mathrm{L}^{\infty}}\right) \\
& \leq \omega(\Theta|s-t|) .
\end{aligned}
$$

Hence, (3.2g) yields the estimate

$$
\left\|\partial_{e} \widetilde{W}\left(t, \theta_{\mathrm{appl}}(s)\right)-\partial_{e} \widetilde{W}\left(t, \theta_{\mathrm{appl}}(t)\right)\right\|_{\mathrm{L}^{2}}^{2} \leq C_{1}^{e}\left(\int_{\Omega} \widetilde{W}\left(t, \theta_{\mathrm{appl}}(t)\right) \mathrm{d} x+C_{0}^{e}|\Omega|\right) \bar{\omega}_{s, t},
$$

which implies thanks to (3.11) that

$$
\left\|\partial_{e} \widetilde{W}\left(t, \theta_{\mathrm{appl}}(s)\right)-\partial_{e} \widetilde{W}\left(t, \theta_{\mathrm{appl}}(t)\right)\right\|_{\mathrm{L}^{2}}^{2} \leq C_{1}^{e}\left(\rho(E)+C_{0}^{e}|\Omega|\right) \bar{\omega}_{s, t} .
$$

Carrying (3.16) into (3.14), and observing that $\mathbf{e}\left(\dot{u}_{\operatorname{Dir}}(\cdot)\right) \in \mathrm{C}^{0}\left([0, T] ; \mathrm{L}^{2}\left(\Omega ; \mathbb{R}_{\mathrm{sym}}^{d \times d}\right)\right)$, one deduces that there exists $\widetilde{C}_{2}^{E}>0$ such that

$$
\mathcal{I}_{\text {var }}^{e}(s, t) \leq \widetilde{C}_{2}^{E}\left(\left\|\mathbf{e}\left(u_{\operatorname{Dir}}(s)\right)-\mathbf{e}\left(u_{\operatorname{Dir}}(t)\right)\right\|_{\mathrm{L}^{2}}+\sqrt{\bar{\omega}_{s, t}}\right) .
$$

Finally, we insert (3.13) and (3.17) in (3.12) and obtain

$$
\begin{aligned}
\mathcal{I}_{e}(s, t) \leq & \widetilde{C}_{1}^{E}\left\|\mathbf{e}\left(\dot{u}_{\operatorname{Dir}}(s)\right)-\mathbf{e}\left(\dot{u}_{\operatorname{Dir}}(t)\right)\right\|_{\mathrm{L}^{2}} \\
& +\widetilde{C}_{2}^{E}\left(\left\|\mathbf{e}\left(u_{\operatorname{Dir}}(s)\right)-\mathbf{e}\left(u_{\operatorname{Dir}}(t)\right)\right\|_{\mathrm{L}^{2}}+\sqrt{\bar{\omega}_{s, t}}\right) .
\end{aligned}
$$

Using the same decomposition for $\mathcal{I}_{\theta}$ as for $\mathcal{I}_{e}$, we have

$$
\mathcal{I}_{\theta}(s, t) \leq \int_{\Omega}\left|\partial_{\theta} \widetilde{W}\left(s, \theta_{\mathrm{appl}}(s)\right)\left(\dot{\theta}_{\mathrm{appl}}(s)-\dot{\theta}_{\mathrm{appl}}(t)\right)\right| \mathrm{d} x+\mathcal{I}_{\mathrm{var}}^{\theta}(s, t)
$$

where

$$
\begin{aligned}
\mathcal{I}_{\text {var }}^{\theta}(s, t) \stackrel{\text { def }}{=} & \int_{\Omega}\left|\left(\partial_{\theta} \widetilde{W}\left(s, \theta_{\text {appl }}(s)\right)-\partial_{\theta} \widetilde{W}\left(t, \theta_{\text {appl }}(s)\right)\right) \dot{\theta}_{\text {appl }}(t)\right| \mathrm{d} x \\
& +\int_{\Omega}\left|\left(\partial_{\theta} \widetilde{W}\left(t, \theta_{\text {appl }}(s)\right)-\partial_{\theta} \widetilde{W}\left(t, \theta_{\text {appl }}(t)\right)\right) \dot{\theta}_{\text {appl }}(t)\right| \mathrm{d} x .
\end{aligned}
$$

Using (3.2e), the first term on the right-hand side of (3.19) can be estimated as follows:

$$
\begin{aligned}
& \int_{\Omega}\left|\partial_{\theta} \widetilde{W}\left(s, \theta_{\text {appl }}(s)\right)\left(\dot{\theta}_{\text {appl }}(s)-\dot{\theta}_{\text {appl }}(t)\right)\right| \mathrm{d} x \\
& \leq\left(C_{1}^{W} \int_{\Omega} \widetilde{W}\left(s, \theta_{\text {appl }}(s)\right) \mathrm{d} x+C_{1}^{W} C_{0}^{W}|\Omega|\right)\left\|\dot{\theta}_{\text {appl }}(s)-\dot{\theta}_{\text {appl }}(t)\right\|_{\mathrm{L}^{\infty}}
\end{aligned}
$$


which implies using once again (3.11) that there exists $\widetilde{C}_{3}^{E}>0$ such that

$$
\int_{\Omega}\left|\partial_{\theta} \widetilde{W}\left(s, \theta_{\text {appl }}(s)\right)\left(\dot{\theta}_{\text {appl }}(s)-\dot{\theta}_{\text {appl }}(t)\right)\right| \mathrm{d} x \leq \widetilde{C}_{3}^{E}\left\|\dot{\theta}_{\text {appl }}(s)-\dot{\theta}_{\text {appl }}(t)\right\|_{\mathrm{L}^{\infty}} .
$$

To estimate $\mathcal{I}_{\text {var }}^{\theta}$ we first deduce from (3.2f), (3.2h), Cauchy-Schwarz's inequality, and (3.15) that

$$
\begin{aligned}
\mathcal{I}_{\text {var }}^{\theta}(s, t) \leq & \Theta\left(C^{\theta}\left\|1+\left|\mathbf{e}\left(u_{\text {Dir }}(s)\right)+\mathbf{e}\left(u_{\text {Dir }}(t)\right)+2 \mathbf{e}(u)\right|+2|z|\right\|_{\mathrm{L}^{2}}\right. \\
& \left\|\mathbf{e}\left(u_{\text {Dir }}(s)\right)-\mathbf{e}\left(u_{\text {Dir }}(t)\right)\right\|_{\mathrm{L}^{2}} \\
& \left.+C_{1}^{\theta}\left(\int_{\Omega} \widetilde{W}\left(t, \theta_{\text {appl }}(t)\right) \mathrm{d} x+C_{0}^{\theta}|\Omega|\right) \bar{\omega}_{s, t}\right) .
\end{aligned}
$$

With Cauchy-Schwarz's inequality, (3.5), $(2.3)$, and $\mathbf{e}\left(u_{\operatorname{Dir}}(\cdot)\right) \in \mathrm{C}^{0}\left([0, T] ; \mathrm{L}^{2}\left(\Omega ; \mathbb{R}_{\text {sym }}^{d \times d}\right)\right)$ we infer that $\left\|1+\left|\mathbf{e}\left(u_{\operatorname{Dir}}(s)\right)+\mathbf{e}\left(u_{\operatorname{Dir}}(t)\right)+2 \mathbf{e}(u)\right|+2|z|\right\|_{\mathrm{L}^{2}}$ is bounded independently of $t, s$, and $q$. Hence, using (3.11), we deduce that there exists $\widetilde{C}_{4}^{E}>0$ such that

$$
\mathcal{I}_{\mathrm{var}}^{\theta}(s, t) \leq \widetilde{C}_{4}^{E}\left(\left\|\mathbf{e}\left(u_{\operatorname{Dir}}(s)\right)-\mathbf{e}\left(u_{\operatorname{Dir}}(t)\right)\right\|_{\mathrm{L}^{2}}+\bar{\omega}_{s, t}\right) .
$$

Carrying (3.20) and (3.22) into (3.19), we obtain

$$
\mathcal{I}_{\theta}(s, t) \leq \widetilde{C}_{3}^{E}\left\|\dot{\theta}_{\mathrm{appl}}(s)-\dot{\theta}_{\mathrm{appl}}(t)\right\|_{\mathrm{L}^{\infty}}+\widetilde{C}_{4}^{E}\left(\left\|\mathbf{e}\left(u_{\mathrm{Dir}}(s)\right)-\mathbf{e}\left(u_{\mathrm{Dir}}(t)\right)\right\|_{\mathrm{L}^{2}}+\bar{\omega}_{s, t}\right) .
$$

Recalling that (3.1) assumes that $\theta_{\text {appl }}, l$, and $u_{\text {Dir }}$ are $\mathrm{C}^{1}$, the compactness of $[0, T]$ implies uniform continuity of the derivatives. Hence, (3.10d), (3.18), (3.23), and $\bar{\omega}_{s, t} \leq$ $\omega(\Theta|s-t|)$ lead to the existence of a nondecreasing function $\omega^{E}:[0, \infty) \rightarrow[0, \infty)$ with $\omega^{E}(\tau) \rightarrow 0$ for $\tau \searrow 0$ such that

$$
\left|\partial_{t} \mathcal{E}(s, q)-\partial_{t} \mathcal{E}(t, q)\right| \leq \omega^{E}(|s-t|),
$$

whenever $\mathcal{E}(0, q) \leq E$. This concludes the proof.

Next we introduce the set of stable states defined as follows:

$$
\mathcal{S}(t) \stackrel{\text { def }}{=}\{q \in \mathcal{Q} \mid \forall \bar{q} \in \mathcal{Q}: \mathcal{E}(t, q) \leq \mathcal{E}(t, \bar{q})+\mathcal{D}(z, \bar{z})\}
$$

Let us observe that $(\mathrm{S})$ is equivalent to $q(t)=(u(t), z(t)) \in \mathcal{S}(t)$.

Lemma 3.4. Let the assumptions (3.1), (3.2a) to (3.2e), (3.2j), (2.7), and (3.3) hold. If $t_{n} \rightarrow t_{*}, z_{n} \rightarrow z_{*}, q_{n}=\left(u_{n}, z_{n}\right) \in \mathcal{S}\left(t_{n}\right)$, and $\sup _{n \in \mathbb{N}} \mathcal{E}\left(t_{n}, q_{n}\right)<\infty$, then

$$
\mathcal{E}\left(t_{n}, q_{n}\right) \rightarrow \mathcal{E}\left(t_{*}, q_{*}\right) \quad \text { and } \quad q_{n} \rightarrow q_{*}=\left(u_{*}, z_{*}\right) \text { in } \mathcal{Q} \text { strongly }
$$

where $u_{*}=\operatorname{Argmin} \mathcal{E}\left(t_{*}, \cdot, z_{*}\right)$.

Proof. We recall that by $(3.2 \mathrm{a})$ the functional $\mathcal{F} \ni u \mapsto \mathcal{E}(t, u, z)$ is strictly convex. Hence, by weak lower semicontinuity there is for each pair $(t, z) \in[0, T] \times \mathcal{Z}$ a unique minimizer $u=U(t, z)$.

We first use the coercivity (3.5) to see that the sequence $\left(u_{n}\right)_{n \in \mathbb{N}}$ is bounded in $\mathcal{F}$. Thus, there exist a convergent subsequence $\left(u_{n_{j}}\right)_{j \in \mathbb{N}}$ and $\widetilde{u}$ with $q_{n_{j}} \rightarrow \widetilde{q}=$ $\left(\widetilde{u}, z_{*}\right)$ for $j \rightarrow \infty$. Since $\mathcal{E}$ is weakly lower semicontinuous, we infer $\mathcal{E}\left(t_{*}, \widetilde{q}\right) \leq$ $\liminf _{j \rightarrow \infty} \mathcal{E}\left(t_{n_{j}}, q_{n_{j}}\right)$. 
Using the stability of $q_{n}$ and testing with $q_{*}=\left(u_{*}, z_{*}\right)$ we have

$$
\begin{aligned}
\mathcal{E}\left(t_{n}, q_{n}\right) & \leq \mathcal{E}\left(t_{n}, q_{*}\right)+\mathcal{D}\left(z_{n}, z_{*}\right) \\
& \leq \mathcal{E}\left(t_{*}, q_{*}\right)+\left(\exp \left(C_{1}^{\mathcal{E}}\left|t_{n}-t_{*}\right|\right)-1\right)\left(\mathcal{E}\left(t_{*}, q_{*}\right)+C_{0}^{\mathcal{E}}\right)+\mathcal{D}\left(z_{n}, z_{*}\right) .
\end{aligned}
$$

Passing to the limit $n \rightarrow \infty$ gives $\lim \sup _{n \rightarrow \infty} \mathcal{E}\left(t_{n}, q_{n}\right) \leq \mathcal{E}\left(t_{*}, q_{*}\right)$. Since $u_{*}$ is the unique minimizer, we have

$$
\mathcal{E}\left(t_{*}, q_{*}\right) \leq \mathcal{E}\left(t_{*}, \widetilde{u}, z_{*}\right) \leq \liminf _{j \rightarrow \infty} \mathcal{E}\left(t_{n_{j}}, q_{n_{j}}\right) \leq \limsup _{n \rightarrow \infty} \mathcal{E}\left(t_{n}, q_{n}\right) \leq \mathcal{E}\left(t_{*}, q_{*}\right) .
$$

Thus, we conclude that $\mathcal{E}\left(t_{n}, q_{n}\right) \rightarrow \mathcal{E}\left(t_{*}, q_{*}\right)$ and that $\widetilde{u}$ is equal to the unique minimizer $u_{*}$. This also shows that the whole sequence converges: $u_{n} \rightarrow u_{*}$.

It remains to show that the convergence must in fact be strong, which will follow from the crucial property that the integrand of $(e, z, A) \mapsto W(e, z, \theta)+\frac{\sigma}{2}|A|^{2}$ is strictly convex in $(e, A)$. First we employ (3.7) to conclude that we also have $\mathcal{E}\left(t_{*}, q_{n}\right) \rightarrow \mathcal{E}\left(t_{*}, q_{*}\right)$, since $\mathcal{E}\left(t_{n}, q_{n}\right)-\mathcal{E}\left(t_{*}, q_{n}\right)$ can be estimated via $C\left|t_{n}-t_{*}\right|$. Next observe that $\mathcal{E}\left(t_{*}, \cdot\right)$ is the sum of the two weakly lower semicontinuous functionals $\mathcal{I}_{1}: q \mapsto \int_{\Omega} W\left(\mathbf{e}\left(u+u_{\text {Dir }}\left(t_{*}\right)\right), z, \theta_{\text {appl }}\left(t_{*}\right)\right) \mathrm{d} x$ and $\mathcal{I}_{2}: q \mapsto \int_{\Omega} \frac{\sigma}{2}|\nabla z|^{2} \mathrm{~d} x$ and the linear functional $-\left\langle l\left(t_{*}\right), \cdot\right\rangle$. Thus, we have $\mathcal{I}_{k}\left(q_{n}\right) \rightarrow \mathcal{I}_{k}\left(q_{*}\right)$ for $k=1,2$. The case $k=2$ yields $z_{n} \rightarrow z_{*}$ in $\mathrm{H}^{1}\left(\Omega ; \mathbb{R}^{N}\right)$ strongly, since in Hilbert spaces weak convergence plus convergence of the norms implies strong convergence.

To establish strong convergence of the $u$-component, we introduce $\widehat{q}_{n}=\left(u_{n}, z_{*}\right)$ and employ condition $(3.2 \mathrm{j})$ to obtain

$$
\begin{aligned}
\left|\mathcal{I}_{1}\left(\widehat{q}_{n}\right)-\mathcal{I}_{1}\left(q_{n}\right)\right| & \leq \int_{\Omega} C\left(1+\left|\mathbf{e}\left(u_{n}+u_{\text {Dir }}\left(t_{*}\right)\right)\right|\right)^{\widehat{p}} \omega\left(\left|z_{n}(x)-z_{*}(x)\right|\right) \mathrm{d} x \\
& \leq C 2^{\widehat{p} / 2}\left(|\Omega|+\left\|\mathbf{e}\left(u_{n}+u_{\text {Dir }}\left(t_{*}\right)\right)\right\|_{\mathrm{L}^{2}}^{2}\right)^{\widehat{p} / 2}\left\|\omega\left(\left|z_{n}-z_{*}\right|\right)\right\|_{\mathrm{L}^{\bar{p}}}
\end{aligned}
$$

where we used Hölder's inequality with $\bar{p}=2 /(2-\widehat{p}) \in(1, \infty)$. In $(3.26)$ the first factor is bounded because of weak convergence. The second factor $\left\|\omega\left(\left|z_{n}-z_{*}\right|\right)\right\|_{\mathrm{L}^{\bar{p}}}$ converges to 0 , since $\omega\left(\left|z_{n}-z_{*}\right|\right)$ is uniformly bounded by $\omega(\operatorname{diam}(Z))$ and since $\left|z_{n}-z_{*}\right| \rightarrow 0$ in $\mathrm{L}^{2}(\Omega)$. Thus, we conclude

$$
\left|\mathcal{I}_{1}\left(\widehat{q}_{n}\right)-\mathcal{I}_{1}\left(q_{*}\right)\right| \leq\left|\mathcal{I}_{1}\left(\widehat{q}_{n}\right)-\mathcal{I}_{1}\left(q_{n}\right)\right|+\left|\mathcal{I}_{1}\left(q_{n}\right)-\mathcal{I}_{1}\left(q_{*}\right)\right| \rightarrow 0 .
$$

In $\mathcal{I}_{1}\left(\widehat{q}_{n}\right)$ the integrand is $x \mapsto W\left(x, \mathbf{e}\left(u_{n}(x)+u_{\text {Dir }}\left(t_{*}, x\right)\right), z_{*}(x), \theta\left(t_{*}, x\right)\right)$, where $W(x, \cdot, z, \theta): \mathbb{R}_{\mathrm{sym}}^{d \times d} \rightarrow \mathbb{R}$ is strictly convex and we can apply [Vis84, Thm. 3] to conclude $\mathbf{e}\left(u_{n}\right) \rightarrow \mathbf{e}\left(u_{*}\right)$ in $\mathrm{L}^{2}\left(\Omega ; \mathbb{R}_{\mathrm{sym}}^{d \times d}\right)$ strongly, which means $u_{n} \rightarrow u_{*}$ in $\mathrm{H}^{1}\left(\Omega ; \mathbb{R}^{d}\right)$ strongly.

The assumptions on the prescribed temperature profile $\theta_{\text {appl }}$, the external loading $l$, the Dirichlet boundary condition $u_{\text {Dir }}$, and the stored energy density $W$ given above allow us to prove that the power $\partial_{t} \mathcal{E}(t, q)$ is locally Lipschitz continuous with respect to $q$ uniformly with respect to $t$. This property will play a key role in the proof of the Lipschitz continuity of energetic solutions, which will be established in the next section.

Lemma 3.5. Assume (3.1), (3.2b) to (3.2e), (3.2h), and (3.2i) hold. Then, for all $R>0$ there exists a constant $C_{R}>0$ such that

$$
\begin{aligned}
& \forall t \in[0, T] \forall q_{1}, q_{2} \in \mathcal{Q} \text { with }\left\|q_{1}\right\|_{\mathcal{Q}},\left\|q_{2}\right\|_{\mathcal{Q}} \leq R: \\
& \left|\partial_{t} \mathcal{E}\left(t, q_{1}\right)-\partial_{t} \mathcal{E}\left(t, q_{2}\right)\right| \leq C_{R}\left\|q_{1}-q_{2}\right\|_{\mathcal{Q}} .
\end{aligned}
$$


Proof. We let $e_{i} \stackrel{\text { def }}{=} \mathbf{e}\left(u_{i}+u_{\text {Dir }}(t)\right)$ and $\widetilde{W}\left(e_{i}, z_{i}\right) \stackrel{\text { def }}{=} W\left(\mathbf{e}\left(u_{i}+u_{\text {Dir }}(t)\right), z_{i}, \theta_{\text {appl }}(t)\right)$ for $i=1,2$. Recalling $\eta \stackrel{\text { def }}{=}\left\|\mathbf{e}\left(\dot{u}_{\text {Dir }}(\cdot)\right)\right\|_{\mathrm{C}^{0}\left([0, T] ; \mathrm{L}^{2}\right)}$ and $\Theta \stackrel{\text { def }}{=}\left\|\dot{\theta}_{\text {appl }}(\cdot)\right\|_{\mathrm{C}^{0}\left([0, T] ; \mathrm{L}^{\infty}\right)}$ and using Cauchy-Schwarz's and Hölder's inequalities we infer that

$$
\begin{aligned}
\left|\partial_{t} \mathcal{E}\left(t, q_{1}\right)-\partial_{t} \mathcal{E}\left(t, q_{2}\right)\right| \leq & \eta\left(\int_{\Omega}\left|\partial_{e} \widetilde{W}\left(e_{1}, z_{1}\right)-\partial_{e} \widetilde{W}\left(e_{2}, z_{2}\right)\right|^{2} \mathrm{~d} x\right)^{1 / 2} \\
& +\Theta \int_{\Omega}\left|\partial_{\theta} \widetilde{W}\left(e_{1}, z_{1}\right)-\partial_{\theta} \widetilde{W}\left(e_{2}, z_{2}\right)\right| \mathrm{d} x \\
& +\|\dot{i}(t)\|_{\left(\mathrm{H}^{1}\right)^{\prime}}\left\|u_{2}-u_{1}\right\|_{\mathrm{H}^{1}},
\end{aligned}
$$

which, using (3.2h) and (3.2i), implies

$$
\begin{aligned}
\left|\partial_{t} \mathcal{E}\left(t, q_{1}\right)-\partial_{t} \mathcal{E}\left(t, q_{2}\right)\right| \leq & C^{\theta} \Theta\left(\int_{\Omega}\left(\left|e_{1}-e_{2}\right|+\left|z_{1}-z_{2}\right|\right)\left(1+\left|e_{1}+e_{2}\right|+\left|z_{1}+z_{2}\right|\right) \mathrm{d} x\right) \\
& +C^{e} \eta\left(\int_{\Omega}\left(\left|e_{1}-e_{2}\right|+\left|z_{1}-z_{2}\right|\right)^{2} \mathrm{~d} x\right)^{1 / 2} \\
& +\|\dot{l}(t)\|_{\left(\mathrm{H}^{1}\right)^{\prime}}\left\|u_{2}-u_{1}\right\|_{\mathrm{H}^{1}} . \\
\leq & \left(C^{e} \eta+C^{\theta} \Theta K\left(t, q_{1}, q_{2}\right)\right)\left(\left\|u_{1}-u_{2}\right\|_{\mathrm{H}^{1}}+\left\|z_{1}-z_{2}\right\|_{\mathrm{L}^{2}}\right) \\
& +\|\dot{i}(t)\|_{\left(\mathrm{H}^{1}\right)^{\prime}}\left\|u_{2}-u_{1}\right\|_{\mathrm{H}^{1}},
\end{aligned}
$$

where $K\left(t, q_{1}, q_{2}\right) \stackrel{\text { def }}{=} \sqrt{|\Omega|}+\left\|z_{1}\right\|_{\mathrm{L}^{2}}+\left\|z_{2}\right\|_{\mathrm{L}^{2}}+\left\|u_{1}\right\|_{\mathrm{H}^{1}}+\left\|u_{2}\right\|_{\mathrm{H}^{1}}+2\left\|u_{\text {Dir }}(t)\right\|_{\mathrm{H}^{1}}$. Using (3.1) the desired estimate is established.

4. Temporal regularity via uniform convexity. In this section we study a better case, where $\mathcal{E}(t, \cdot)$ is uniformly convex and $\mathcal{D}\left(z_{0}, z_{1}\right)$ depends only on the difference $z_{1}-z_{0}$. The arguments follow the method developed in [MiT04, sect. 7]; see also [MiR07].

We assume that $W$ is $\alpha_{W}$-uniformly convex jointly in the first two arguments; namely, there exists a modulus of convexity $\alpha_{W}>0$ such that for all $e_{1}, e_{2} \in \mathbb{R}_{\mathrm{sym}}^{d \times d}$, $z_{1}, z_{2} \in Z$, and $\lambda \in[0,1]$ we have

$$
\begin{aligned}
\forall \theta \in\left[\theta_{\min }, \theta_{\max }\right]: W\left(e_{\lambda}, z_{\lambda}, \theta\right) \leq & (1-\lambda) W\left(e_{1}, z_{1}, \theta\right)+\lambda W\left(e_{2}, z_{2}, \theta\right) \\
& -\frac{\alpha_{W}}{2} \lambda(1-\lambda)\left(\left|e_{2}-e_{1}\right|^{2}+\left|z_{2}-z_{1}\right|^{2}\right),
\end{aligned}
$$

where $e_{\lambda} \stackrel{\text { def }}{=}(1-\lambda) e_{1}+\lambda e_{2}$ and $z_{\lambda} \stackrel{\text { def }}{=}(1-\lambda) z_{1}+\lambda z_{2}$. With $q_{\lambda} \stackrel{\text { def }}{=}(1-\lambda) q_{1}+\lambda q_{2}$, we have

$$
\mathcal{E}\left(t, q_{\lambda}\right) \leq(1-\lambda) \mathcal{E}\left(t, q_{1}\right)+\lambda \mathcal{E}\left(t, q_{2}\right)-\frac{\widehat{\kappa}}{2} \lambda(1-\lambda)\left\|q_{2}-q_{1}\right\|_{\mathcal{B}}^{2},
$$

where $\widehat{\kappa} \stackrel{\text { def }}{=} \min \left(\alpha_{W}, \sigma\right)$ and $\|q\|_{\mathcal{B}}^{2} \stackrel{\text { def }}{=}\|\mathbf{e}(u)\|_{\mathrm{L}^{2}}^{2}+\|z\|_{\mathrm{H}^{1}}^{2}$. Using Korn's inequality (2.2), we find $\|q\|_{\mathcal{B}}^{2} \geq \min \left(c_{\text {Korn }}, 1\right)\|q\|_{\mathcal{Q}}^{2}$. Hence, we deduce

$$
\begin{aligned}
& \forall q_{1}, q_{2} \in \mathcal{Q} \forall t \in[0, T] \forall \lambda \in[0,1]: \\
& \mathcal{E}\left(t, q_{\lambda}\right) \leq(1-\lambda) \mathcal{E}\left(t, q_{1}\right)+\lambda \mathcal{E}\left(t, q_{2}\right)-\frac{\kappa}{2} \lambda(1-\lambda)\left\|q_{2}-q_{1}\right\|_{\mathcal{Q}}^{2},
\end{aligned}
$$

where $\kappa=\widehat{\kappa} \min \left(c_{\text {Korn }}, 1\right)$. In other words, $\mathcal{E}(t, \cdot)$ is $\kappa$-uniformly convex on $\mathcal{Q}$. 
The next result establishes that in the present setting energetic solutions are Lipschitz continuous in time, which essentially relies on the uniform convexity (4.2) of $\mathcal{E}(t, \cdot)$ and on assumption (2.6) for the dissipation $D$ which implies the convexity of the dissipation distance $\mathcal{D}(q, \cdot): \mathcal{Q} \rightarrow[0, \infty]$.

Notice that the dissipation distance is called translation invariant if $D$ satisfies (2.6). Then, $\mathcal{D}\left(z_{0}, z_{1}\right)=\Psi\left(z_{1}-z_{0}\right)$ with $\Psi(v)=\int_{\Omega} \psi(v(x)) \mathrm{d} x$ and $\Psi$ plays the role of a (possible unsymmetric) $\mathrm{L}^{1}$ norm.

TheOrem 4.1 (Lipschitz continuity). Assume that (2.6), (2.7), (3.1), (3.2b) to (3.2e), (3.2h), (3.2i), (3.3), and (4.1) hold. Then, any energetic solution $q$ is Lipschitz continuous. More precisely, let $R \stackrel{\text { def }}{=}\|q\|_{\mathrm{L}^{\infty}([0, T] ; \mathcal{Q})}$ and $C_{R}>0$ be given by Lemma 3.5; then $\|\dot{q}(t)\|_{\mathcal{Q}} \leq \frac{2 C_{R}}{\kappa}$ for a.e. $t \in[0, T]$ with $\kappa$ from $(4.2)$.

Proof. We first prove that uniform convexity allows us to improve the stability (S) into the following stronger statement:

$$
\forall s \in[0, T] \forall \widehat{q} \in \mathcal{Q}: \mathcal{E}(s, q(s))+\frac{\kappa}{2}\|\widehat{q}-q(s)\|_{\mathcal{Q}}^{2} \leq \mathcal{E}(s, \widehat{q})+\Psi(\widehat{q}-q(s)) .
$$

Indeed, fix $s \in[0, T]$ and define the functional $\mathcal{J}$ via $\mathcal{J}(\widehat{q})=\mathcal{E}(s, \widehat{q})+\Psi(\widehat{q}-q(s))$ for all $\widehat{q} \in \mathcal{Q}$. Since $q$ is an energetic solution and hence satisfies $(\mathrm{S})$, we know that $q(s)$ is a global minimizer of $\mathcal{J}$. Moreover, since $\Psi$ is convex we obtain that $\mathcal{J}$ is $\kappa$-uniformly convex on $\mathcal{Q}$ by using (4.2). Thus, for $\widehat{q} \in \mathcal{Q}$ and $\lambda \in(0,1)$ we let $q_{\lambda}=(1-\lambda) \widehat{q}+\lambda q(s)$ and obtain

$$
\begin{aligned}
\mathcal{J}(q(s))+\frac{\kappa}{2} \lambda(1-\lambda)\|\widehat{q}-q(s)\|_{\mathcal{Q}}^{2} & \leq \mathcal{J}\left(q_{\lambda}\right)+\frac{\kappa}{2} \lambda(1-\lambda)\|\widehat{q}-q(s)\|_{\mathcal{Q}}^{2} \\
& \leq(1-\lambda) \mathcal{J}(\widehat{q})+\lambda \mathcal{J}(q(s)) .
\end{aligned}
$$

Subtracting $\lambda \mathcal{J}(q(s))$ and dividing by $(1-\lambda)$ gives $\mathcal{J}(q(s))+\frac{\kappa}{2} \lambda\|\widehat{q}-q(s)\|_{\mathcal{Q}}^{2} \leq \mathcal{J}(\widehat{q})$. Now the definition of $\mathcal{J}$ and the limit $\lambda \rightarrow 1$ lead to the desired estimate (4.3). Hence, for all $s, t \in[0, T]$ such that $s \leq t$, by choosing $\widehat{q}=q(t)$ we get

$$
\begin{aligned}
& \frac{\kappa}{2}\|q(t)-q(s)\|_{\mathcal{Q}}^{2} \leq \mathcal{E}(s, q(t))-\mathcal{E}(s, q(s))+\mathcal{D}(z(s), z(t)) \\
& \leq \mathcal{E}(s, q(t))-\mathcal{E}(s, q(s))+\operatorname{Var}_{\mathcal{D}}(z,[s, t]) \\
& =-\int_{s}^{t} \partial_{r} \mathcal{E}(r, q(t)) \mathrm{d} r+\int_{s}^{t} \partial_{r} \mathcal{E}(r, q(r)) \mathrm{d} r \leq C_{R} \int_{s}^{t}\|q(r)-q(t)\|_{\mathcal{Q}} \mathrm{d} r
\end{aligned}
$$

The second estimate comes from the definition of $\operatorname{Var}_{\mathcal{D}}$, the third identity follows from the energy identity (E) and the additivity property of the dissipation, i.e.,

$$
\operatorname{Var}_{\mathcal{D}}(z,[0, t])=\operatorname{Var}_{\mathcal{D}}(z,[0, s])+\operatorname{Var}_{\mathcal{D}}(z,[s, t])
$$

and the last one results from (3.27). We conclude by applying Lemma 4.2.

Lemma 4.2. Let $q \in \mathrm{L}^{\infty}([0, T] ; \mathcal{Q})$ and $C>0$ be given such that, for all $s, t \in$ $[0, T]$ such that $s \leq t$, we have

$$
\frac{\kappa}{2}\|q(t)-q(s)\|_{\mathcal{Q}}^{2} \leq C \int_{s}^{t}\|q(r)-q(t)\|_{\mathcal{Q}} \mathrm{d} r .
$$

Then, $q \in \mathrm{C}^{\operatorname{Lip}}([0, T] ; \mathcal{Q})$ with $\|\dot{q}(t)\|_{\mathcal{Q}} \leq \frac{2 C}{\kappa}$ for a.e. $t \in[0, T]$.

The proof is a simple adaptation of the proof of Theorem 7.5 in [MiT04]. 
5. Convergence of the space-time discretization. In this section we treat the question of convergence of spatially and temporally discretized problems. As we do not have uniqueness of solutions for the full problem, we cannot expect convergence of the whole approximation sequence. But, as in the existence theorem (Theorem 3.1), we will obtain convergence of subsequences to solutions of the full problem. The approach here follows the abstract $\Gamma$-convergence theory developed in [MRS08] and the specialization to general numerical approaches in [MiR06]. However, for the special model at hand, we can show more than is stated in the above-mentioned general papers. Hence, we provide a full independent proof here.

For the time discretization we consider $\tau \in(0, T)$ and a partition $\Pi^{\tau}=\left\{0=t_{0}^{\tau}<\right.$ $\left.t_{1}^{\tau}<\cdots<t_{k^{\tau}}^{\tau}=T\right\}$ with

$$
t_{k}^{\tau}-t_{k-1}^{\tau} \leq \tau \text { for } k=1, \ldots, k^{\tau}
$$

In particular, we do not assume our partitions to be equidistant.

For the spatial discretization we choose a set of length parameters $h>0$ accumulating at $h=0$ and let $\mathcal{F}_{h}$ and $V_{h}$ be closed subspaces of $\mathcal{F}$ and $V=\mathrm{H}^{1}\left(\Omega ; \mathbb{R}^{N}\right)$, respectively. Typically, $\mathcal{F}_{h}$ and $V_{h}$ are finite-dimensional subspaces of $\mathcal{F}$ and $V$, like finite element spaces or Galerkin subspaces. We let $\mathcal{Q}_{h} \stackrel{\text { def }}{=} \mathcal{F}_{h} \times \mathcal{Z}_{h}$, with $\mathcal{Z}_{h}=\left\{z_{h} \in V_{h} \mid z_{h}(x) \in Z\right.$ a.e. in $\left.\Omega\right\}=\mathcal{Z} \cap V_{h}$. We assume that the sets $\mathcal{Q}_{h}$ satisfy the standard density assumption:

$$
\forall q \in \mathcal{Q} \exists\left(q_{h}\right)_{h>0}: q_{h} \in \mathcal{Q}_{h} \text { and } q_{h} \rightarrow q \text { strongly in } \mathcal{Q} \text {. }
$$

By convention, let $\mathcal{Q}_{0} \stackrel{\text { def }}{=} \mathcal{F}_{0} \times \mathcal{Z}_{0}=\mathcal{F} \times \mathcal{Z}$.

To have some specific spatial discretization in mind, we may assume that $\Omega$ is a polyhedral domain and that $\Gamma_{\text {Dir }} \subset \partial \Omega$ is a finite union of faces of $\Omega$. Then, for each $h>0$ choose a triangulation $\mathcal{T}_{h}$ of $\Omega$, such that all edges have at most length $h$. Now, let $V_{h}$ be the space of functions that are affine on each polyhedron of $\mathcal{T}_{h}$. Hence $V_{h} \subset \mathrm{H}^{1}(\Omega)$, and we let $\mathcal{F}_{h}=V_{h} \cap \mathcal{F}$ and $\mathcal{Z}_{h}=V_{h} \cap \mathcal{Z}$. It is then standard in finite element theory to show the density property (5.1).

We approximate the initial condition $q_{0}$ by $\left[q_{0}\right]^{h} \in \mathcal{Q}_{h}$ and consider the following incremental problems:

$$
(\mathrm{IP})^{\tau, h}\left\{\begin{array}{l}
\text { for } k=1, \ldots, k^{\tau} \text { find } \\
q_{k}^{\tau, h} \stackrel{\text { def }}{=}\left(u_{k}^{\tau, h}, z_{k}^{\tau, h}\right) \in \operatorname{Argmin}\left\{\mathcal{E}\left(t_{k}^{\tau}, \widehat{q}^{h}\right)+\mathcal{D}\left(z_{k-1}^{\tau, h}, \widehat{z}^{h}\right) \mid \widehat{q}^{h} \stackrel{\text { def }}{=}\left(\widehat{u}^{h}, \widehat{z}^{h}\right) \in \mathcal{Q}_{h}\right\} .
\end{array}\right.
$$

We define now the approximate solution $\bar{q}^{\tau, h}:[0, T] \rightarrow \mathcal{Q}$ as the right-continuous piecewise constant approximation, namely

$$
\bar{q}^{\tau, h}(t) \stackrel{\text { def }}{=}\left\{\begin{array}{l}
q_{k-1}^{\tau, h} \text { for } t_{k-1}^{\tau} \leq t<t_{k}^{\tau}, k=1, \ldots, k^{\tau}, \\
q_{k^{\tau}}^{\tau, h} \text { for } t=T .
\end{array}\right.
$$

It is convenient to introduce the set of stable states $\mathcal{S}_{h}(t)$ for any $t \in[0, T]$ by simply replacing $\mathcal{Q}$ by $\mathcal{Q}_{h}$ in (3.24). Observe that if $h=0$, then $\mathcal{S}_{0}(t)=\mathcal{S}(t)$. Moreover, we define $\eta_{k}^{\tau, h} \stackrel{\text { def }}{=} \mathcal{E}\left(t_{k}^{\tau}, q_{k}^{\tau, h}\right)$ and $\delta_{k}^{\tau, h} \stackrel{\text { def }}{=} \mathcal{D}\left(z_{k-1}^{\tau, h}, z_{k}^{\tau, h}\right)$.

The next result, which is fundamental for the energetic approach (cf. [MiT99, MTL02]), shows that the fully implicit incremental minimization problem (IP) is suited perfectly for the energetic formulation (S) and (E). At the time-discrete level we again obtain stability and a two-sided energy estimate. This will allow us to 
derive suitable a priori estimates. The essential feature is that $\mathcal{D}$ satisfies the triangle inequality.

Proposition 5.1. Assume that (2.7), (3.3), (3.1), (3.2b), (3.2c), and $(3.2 \mathrm{~d})$ hold. Then the incremental problems (IP $)^{\tau, h}$ admit a solution $\left(q_{k}^{\tau, h}\right)_{1 \leq k \leq k^{\tau}}$. Moreover, we have

discrete stability: $q_{k}^{\tau, h} \in \mathcal{S}_{h}\left(t_{k}^{\tau}\right)$,

discrete upper energy estimate:

$\forall k \in\left\{1, \ldots, k^{\tau}\right\}: \eta_{k}^{\tau, h}-\eta_{k-1}^{\tau, h}+\delta_{k}^{\tau, h} \leq \int_{t_{k-1}^{\tau}}^{t_{k}^{\tau}} \partial_{t} \mathcal{E}\left(t, q_{k-1}^{\tau, h}\right) \mathrm{d} t$,

discrete lower energy estimate:

$\forall k \in\left\{2, \ldots, k^{\tau}\right\}: \eta_{k}^{\tau, h}-\eta_{k-1}^{\tau, h}+\delta_{k}^{\tau, h} \geq \int_{t_{k-1}^{\tau}}^{t_{k}^{\tau}} \partial_{t} \mathcal{E}\left(t, q_{k}^{\tau, h}\right) \mathrm{d} t$.

Proof. The existence of minimizers in each incremental step is a direct consequence of the coercivity of $\mathcal{E}(t, \cdot): \mathcal{Q} \rightarrow \mathbb{R}$, the nonnegativity of $\mathcal{D}$, and the weak lower semicontinuity of $\mathcal{E}$ and $\mathcal{D}$. Of course, all these properties remain valid if the minimization is restricted to the closed subspace $\mathcal{Q}_{h} \subset \mathcal{Q}$.

For the discrete stability we use first that $q_{k}^{\tau, h}, k=1, \ldots, k^{\tau}$, is a minimizer and that $\mathcal{D}$ satisfies the triangle inequality (see $(2.7 \mathrm{~b}$ ) and integrate over $\Omega$ ): for all $\widehat{q}^{h} \in \mathcal{Q}_{h}$ we have

$$
\mathcal{E}\left(t_{k}^{\tau}, q_{k}^{\tau, h}\right) \leq \mathcal{E}\left(t_{k}^{\tau}, \widehat{q}^{h}\right)+\mathcal{D}\left(z_{k-1}^{\tau, h}, \widehat{z}^{h}\right)-\mathcal{D}\left(z_{k-1}^{\tau, h}, z_{k}^{\tau, h}\right) \leq \mathcal{E}\left(t_{k}^{\tau}, \widehat{q}^{h}\right)+\mathcal{D}\left(z_{k}^{\tau, h}, \widehat{z}^{h}\right)
$$

which yields immediately (5.3). Since $q_{k}^{\tau, h} \in \operatorname{Argmin}\left\{\mathcal{E}\left(t_{k}^{\tau}, \widehat{q}^{h}\right)+\mathcal{D}\left(z_{k-1}^{\tau, h}, \widehat{z}^{h}\right) \mid \widehat{q}^{h} \in\right.$ $\left.\mathcal{Q}_{h}\right\}$ we may choose $\widehat{q}^{h}=q_{k-1}^{\tau, h}$ and find

$$
\eta_{k}^{\tau, h}-\eta_{k-1}^{\tau, h}+\delta_{k}^{\tau, h} \leq \mathcal{E}\left(t_{k}^{\tau}, q_{k-1}^{\tau, h}\right)-\eta_{k-1}^{\tau, h}=\int_{t_{k-1}^{\tau}}^{t_{k}^{\tau}} \partial_{t} \mathcal{E}\left(t, q_{k-1}^{\tau, h}\right) \mathrm{d} t
$$

On the other hand, we rewrite (5.6) for $q_{k-1}^{\tau, h}$, choose $\widehat{q}^{h}=q_{k}^{\tau, h}$, and obtain

$$
\eta_{k}^{\tau, h}-\eta_{k-1}^{\tau, h}+\delta_{k}^{\tau, h} \geq \eta_{k}^{\tau, h}-\mathcal{E}\left(t_{k-1}^{\tau}, q_{k}^{\tau, h}\right)=\int_{t_{k-1}^{\tau}}^{t_{k}^{\tau}} \partial_{t} \mathcal{E}\left(t, q_{k}^{\tau, h}\right) \mathrm{d} t
$$

Thus, (5.4) and (5.5) are established.

To investigate the asymptotics when $\tau$ and $h$ tend to 0 we need a compactness argument suited for the rate-independent case. The following version of Helly's selection principle is a simplified version of the abstract result given in the appendix of [MaM05].

Proposition 5.2 (Helly's selection principle). Let $\mathcal{D}$ be given by (2.8) with $D$ satisfying (2.7) and (3.3). Let $\left(z_{n}\right)_{n \in \mathbb{N}}$ with $z_{n}:[0, T] \rightarrow \mathcal{Z}$ satisfying

$$
\exists C>0 \forall n \in \mathbb{N}: \operatorname{Var}_{\mathcal{D}}\left(z_{n} ;[0, T]\right) \leq C \text { and } \sup _{t \in[0, T]}\left\|z_{n}(t)\right\|_{\mathrm{H}^{1}(\Omega)} \leq C ;
$$


then there exist a subsequence $\left(z_{n_{j}}\right)_{j \in \mathbb{N}}$, a nondecreasing function $\delta:[0, T] \rightarrow \mathbb{R}$, and a limit process $z:[0, T] \rightarrow \mathcal{Z}$ such that for all $s, t \in[0, T]$ with $s \leq t$ we have

$$
\begin{aligned}
& z_{n_{j}}(t) \rightarrow z(t) \text { in } \mathrm{H}^{1}(\Omega), \quad \delta(t)=\lim _{j \rightarrow \infty} \operatorname{Var}_{\mathcal{D}}\left(z_{n_{j}} ;[0, t]\right), \\
& \operatorname{Var}_{\mathcal{D}}(z ;[s, t]) \leq \delta(t)-\delta(s)
\end{aligned}
$$

Our main result states now that the space-time discretization defined via (IP) ${ }^{\tau, h}$ generating the approximants $\bar{q}^{\tau, h}:[0, T] \rightarrow \mathcal{Q}$ has the desirable properties (i) that the sequence of approximants is precompact (which can be understood as the "stability of the numerical algorithm") and (ii) that any limit point of the sequence of approximants is an energetic solution for the rate-independent system $(\mathcal{Q}, \mathcal{E}, \mathcal{D})$ (which can be understood as "consistency of the numerical algorithm"). It should be noted that we do not need to make any assumptions about how the fineness $\tau$ of the partitions or the fineness $h$ of the spatial discretization tend to 0 .

ThEOREM 5.3 (convergence of the approximate solution). Assume that $\mathcal{E}, \mathcal{D}$, and $q_{0}$ satisfy the same assumptions as in Theorem 3.1. Let $\left[q_{0}\right]^{h} \in \mathcal{Q}_{h}$ be such that

$$
\left[q_{0}\right]^{h} \rightarrow q_{0} \text { in } \mathcal{Q}
$$

Then, there exist a subsequence $\left(\tau_{n}, h_{n}\right)_{n \in \mathbb{N}}$ tending to $(0,0)$ and an energetic solution $q:[0, T] \rightarrow \mathcal{Q}$ for $(\mathcal{Q}, \mathcal{E}, \mathcal{D})$ with $q(0)=q_{0}$ and

$$
\begin{aligned}
& u \in \mathrm{L}^{\infty}\left([0, T] ; \mathrm{H}^{1}\left(\Omega ; \mathbb{R}^{d}\right)\right), \\
& z \in \mathrm{L}^{\infty}\left([0, T] ; \mathrm{H}^{1}(\Omega ; Z)\right) \cap \operatorname{BV}\left([0, T] ; \mathrm{L}^{1}(\Omega ; Z)\right),
\end{aligned}
$$

such that for all $t \in[0, T]$ the following convergences hold:

$$
\begin{aligned}
\bar{q}^{\tau_{n}, h_{n}}(t) & \rightarrow q(t) \text { strongly in } \mathcal{Q}, \\
\mathcal{E}\left(t, \bar{q}^{\tau_{n}, h_{n}}(t)\right) & \rightarrow \mathcal{E}(t, q(t)), \\
\operatorname{Var}_{\mathcal{D}}\left(\bar{q}^{\tau_{n}, h_{n}} ;[0, t]\right) & \rightarrow \operatorname{Var}_{\mathcal{D}}(q ;[0, t]) .
\end{aligned}
$$

Proof. The main steps of the proof are similar to those in [MRS08, MiR06], but our energy $\mathcal{E}$ is better behaved, and thus we are able to obtain more precise results. For $t \in[0, T]$ let us introduce the notations

$$
\bar{\eta}^{\tau, h}(t) \stackrel{\text { def }}{=} \mathcal{E}\left(t, \bar{q}^{\tau, h}(t)\right), \eta_{0}^{h} \stackrel{\text { def }}{=} \mathcal{E}\left(0,\left[q_{0}\right]^{h}\right), \bar{\delta}^{\tau, h}(t) \stackrel{\text { def }}{=} \operatorname{Var}_{\mathcal{D}}\left(\bar{z}^{\tau, h} ;[0, t]\right)
$$

and let us recall $\eta_{k}^{\tau, h}=\mathcal{E}\left(t_{k}^{\tau}, q_{k}^{\tau, h}\right)$ and $\delta_{k}^{\tau, h}=\mathcal{D}\left(z_{k-1}^{\tau, h}, z_{k}^{\tau, h}\right)$.

Step 1. A priori estimates. One can observe that (P2) and (3.7) lead to

$$
\forall k \in\left\{1, \ldots, k^{\tau}\right\}: \int_{t_{k-1}^{\tau}}^{t_{k}^{\tau}}\left|\partial_{t} \mathcal{E}\left(t, q_{k-1}^{\tau, h}\right)\right| \mathrm{d} t \leq\left(\exp \left(C_{1}^{\varepsilon}\left(t_{k}^{\tau}-t_{k-1}^{\tau}\right)\right)-1\right)\left(\eta_{k-1}^{\tau, h}+C_{0}^{\varepsilon}\right)
$$

Carrying (5.12) into (5.4), we get

$$
\eta_{k}^{\tau, h}+\delta_{k}^{\tau, h} \leq \exp \left(C_{1}^{\varepsilon}\left(t_{k}^{\tau}-t_{k-1}^{\tau}\right)\right)\left(\eta_{k-1}^{\tau, h}+C_{0}^{\varepsilon}\right)-C_{0}^{\varepsilon},
$$


and observing that $\delta_{k}^{\tau, h} \geq 0$, we obtain by induction

$$
\begin{aligned}
\forall k \in\left\{1, \ldots, k^{\tau}\right\}: \eta_{k}^{\tau, h}+C_{0}^{\varepsilon} & \leq \prod_{j=1}^{k} \exp \left(C_{1}^{\varepsilon}\left(t_{j}^{\tau}-t_{j-1}^{\tau}\right)\right)\left(\eta_{0}^{h}+C_{0}^{\varepsilon}\right) \\
& =\exp \left(C_{1}^{\varepsilon} t_{k}^{\tau}\right)\left(\eta_{0}^{h}+C_{0}^{\varepsilon}\right) .
\end{aligned}
$$

Hence, with (5.2), (3.5), and (3.7), we deduce that

$$
\forall t \in[0, T]:-c_{0} \leq \bar{\eta}^{\tau, h}(t) \leq \exp \left(C_{1}^{\varepsilon} t\right)\left(\eta_{0}^{h}+C_{0}^{\varepsilon}\right)-C_{0}^{\varepsilon}
$$

Next we estimate the dissipated energy $\bar{\delta}^{\tau, h}(t)$ by using (5.13), (5.14), and (3.5): for all $t \in[0, T]$

$$
\begin{aligned}
\bar{\delta}^{\tau, h}(t) & \leq \bar{\delta}^{\tau, h}(T)=\sum_{k=1}^{k^{\tau}} \delta_{k}^{\tau, h} \\
& \leq \eta_{0}^{h}-\eta_{k^{\tau}}^{\tau, h}+\sum_{k=1}^{k^{\tau}}\left(\exp \left(C_{1}^{\varepsilon} t_{k}^{\tau}\right)-\exp \left(C_{1}^{\varepsilon} t_{k-1}^{\tau}\right)\right)\left(\eta_{0}^{h}+C_{0}^{\varepsilon}\right) \\
& \leq \exp \left(C_{1}^{\varepsilon} T\right) \eta_{0}^{h}+\left(c_{0}+\left(\exp \left(C_{1}^{\varepsilon} T\right)-1\right) C_{0}^{\varepsilon}\right) \\
& \leq \exp \left(C_{1}^{\varepsilon} T\right)\left(\eta_{0}^{h}+\max \left(c_{0}, C_{0}^{\varepsilon}\right)\right) .
\end{aligned}
$$

Let us consider now the total variation $\operatorname{Var}\left(\bar{\eta}^{\tau, h} ;[0, T]\right)$ of $\bar{\eta}^{\tau, h}$ on $[0, T]$. Recalling that

$$
\bar{\eta}^{\tau, h}(t)=\mathcal{E}\left(t, \bar{q}^{\tau, h}(t)\right)=\left\{\begin{array}{l}
\mathcal{E}\left(t, q_{k-1}^{\tau, h}\right) \text { for } t_{k-1}^{\tau} \leq t<t_{k}^{\tau}, k=1, \ldots, k^{\tau}, \\
\mathcal{E}\left(T, q_{k^{\tau}}^{\tau, h}\right) \text { for } t=T
\end{array}\right.
$$

we obtain

$$
\begin{aligned}
& \operatorname{Var}\left(\bar{\eta}^{\tau, h} ;[0, T]\right) \leq \sum_{k=1}^{k^{\tau}} \int_{t_{k-1}^{\tau}}^{t_{k}^{\tau}}\left|\partial_{t} \mathcal{E}\left(t, q_{k-1}^{\tau, h}\right)\right| \mathrm{d} t+\sum_{k=1}^{k^{\tau}}\left|\eta_{k}^{\tau, h}-\mathcal{E}\left(t_{k}^{\tau}, q_{k-1}^{\tau, h}\right)\right| \\
& \leq \sum_{k=1}^{k^{\tau}} \int_{t_{k-1}^{\tau}}^{t_{k}^{\tau}}\left|\partial_{t} \mathcal{E}\left(t, q_{k-1}^{\tau, h}\right)\right| \mathrm{d} t+\sum_{k=1}^{k^{\tau}}\left|\eta_{k}^{\tau, h}-\eta_{k-1}^{\tau, h}\right|+\sum_{k=1}^{k^{\tau}} \int_{t_{k-1}^{\tau}}^{t_{k}^{\tau}}\left|\partial_{t} \mathcal{E}\left(t, q_{k-1}^{\tau, h}\right)\right| \mathrm{d} t \\
& =\mathcal{I}_{1}+\mathcal{I}_{2} \quad \text { with } \quad \mathcal{I}_{1} \stackrel{\text { def }}{=} 2 \sum_{k=1}^{k^{\tau}} \int_{t_{k-1}^{\tau}}^{t_{k}^{\tau}}\left|\partial_{t} \mathcal{E}\left(t, q_{k-1}^{\tau, h}\right)\right| \mathrm{d} t \quad \text { and } \quad \mathcal{I}_{2} \stackrel{\text { def }}{=} \sum_{k=1}^{k^{\tau}}\left|\eta_{k}^{\tau, h}-\eta_{k-1}^{\tau, h}\right| .
\end{aligned}
$$

On the one hand, using (5.12), (5.14) and summing for $k=1, \ldots, k^{\tau}$, we obtain

$$
\mathcal{I}_{1} \leq 2 \sum_{k=1}^{k^{\tau}} \exp \left(C_{1}^{\varepsilon}\left(t_{k}^{\tau}-t_{k-1}^{\tau}\right)-1\right)\left(\eta_{k-1}^{\tau, h}+C_{0}^{\varepsilon}\right) \leq 2\left(\exp \left(C_{1}^{\varepsilon} T\right)-1\right)\left(\eta_{0}^{h}+C_{0}^{\varepsilon}\right) .
$$

On the other hand, by (5.4) and (5.5), we have

$$
\begin{aligned}
\mathcal{I}_{2} \leq & \bar{\delta}^{\tau, h}(T)+\sum_{k=1}^{k^{\tau}} \max \left(\int_{t_{k-1}^{\tau}}^{t_{k}^{\tau}}\left|\partial_{t} \mathcal{E}\left(t, q_{k-1}^{\tau, h}\right)\right| \mathrm{d} t, \int_{t_{k-1}^{\tau}}^{t_{k}^{\tau}}\left|\partial_{t} \mathcal{E}\left(t, q_{k}^{\tau, h}\right)\right| \mathrm{d} t\right) \\
& +\max \left(0, \eta_{0}^{h}-\mathcal{E}\left(t_{1}^{\tau}, q_{1}^{\tau, h}\right)\right) .
\end{aligned}
$$


But (P2) and (3.7) lead to

$$
\begin{aligned}
& \int_{t_{k-1}^{\tau}}^{t_{k}^{\tau}}\left|\partial_{t} \mathcal{E}\left(t, q_{k}^{\tau, h}\right)\right| \mathrm{d} t \leq \int_{t_{k-1}^{\tau}}^{t_{k}^{\tau}} C_{1}^{\varepsilon}\left(\mathcal{E}\left(t, q_{k}^{\tau, h}\right)+C_{0}^{\varepsilon}\right) \mathrm{d} t \\
& \leq\left(\exp \left(C_{1}^{\varepsilon}\left(t_{k}^{\tau}-t_{k-1}^{\tau}\right)-1\right)\right)\left(\mathcal{E}\left(t_{k}^{\tau}, q_{k}^{\tau, h}\right)+C_{0}^{\varepsilon}\right) \\
& \leq\left(\exp \left(C_{1}^{\varepsilon}\left(t_{k}^{\tau}-t_{k-1}^{\tau}\right)-1\right) \exp \left(C_{1}^{\varepsilon}\left(t_{k}^{\tau}-t_{k-1}^{\tau}\right)\right)\left(\eta_{k-1}^{\tau, h}+C_{0}^{\varepsilon}\right)\right. \\
& \leq\left(\exp \left(C_{1}^{\varepsilon}\left(t_{k}^{\tau}-t_{k-1}^{\tau}\right)-1\right) \exp \left(C_{1}^{\varepsilon} T\right)\left(\eta_{k-1}^{\tau, h}+C_{0}^{\varepsilon}\right),\right.
\end{aligned}
$$

and thus with (5.12)

$$
\begin{aligned}
& \max \left(\int_{t_{k-1}^{\tau}}^{t_{k}^{\tau}}\left|\partial_{t} \mathcal{E}\left(t, q_{k-1}^{\tau, h}\right)\right| \mathrm{d} t, \int_{t_{k-1}^{\tau}}^{t_{k}^{\tau}}\left|\partial_{t} \mathcal{E}\left(t, q_{k}^{\tau, h}\right)\right| \mathrm{d} t\right) \\
& \leq\left(\exp \left(C_{1}^{\varepsilon}\left(t_{k}^{\tau}-t_{k-1}^{\tau}\right)-1\right) \exp \left(C_{1}^{\varepsilon} T\right)\left(\eta_{k-1}^{\tau, h}+C_{0}^{\varepsilon}\right) .\right.
\end{aligned}
$$

Moreover, we know from (3.5) that $\mathcal{E}\left(t_{1}^{\tau}, q_{1}^{\tau, h}\right) \geq-c_{0}$. Hence, using (5.17), (5.18), and (5.19), we obtain, for all $t \in[0, T]$,

$$
\begin{aligned}
\operatorname{Var}\left(\bar{\eta}^{\tau, h} ;[0, t]\right) \leq \operatorname{Var}\left(\bar{\eta}^{\tau, h} ;[0, T]\right) \leq & \left(\exp \left(C_{1}^{\varepsilon} T\right)-1\right)\left(\eta_{0}^{h}+C_{0}^{\varepsilon}\right)\left(\exp \left(C_{1}^{\varepsilon} T\right)+2\right) \\
& +\bar{\delta}^{\tau, h}(T)+\max \left(0, \eta_{0}^{h}+c_{0}\right)
\end{aligned}
$$

But, with (3.5), we also have

$$
\eta_{0}^{h}=\mathcal{E}\left(0,\left[q_{0}\right]^{h}\right) \leq c_{0}\left\|\left[q_{0}\right]^{h}\right\|_{\mathcal{Q}}^{2}+c_{0},
$$

and since $\left(\left[q_{0}\right]^{h}\right)_{h>0}$ converges to $q_{0}$ in $\mathcal{Q}$, we infer that $\eta_{0}^{h}$ is bounded from above independently of $h$. Hence, (5.15), (5.16), and (5.20) imply that $\left|\bar{\eta}^{\tau, h}(t)\right|, \bar{\delta}^{\tau, h}(t)=$ $\operatorname{Var}_{\mathcal{D}}\left(\bar{z}^{\tau, h} ;[0, t]\right)$, and $\operatorname{Var}\left(\bar{\eta}^{\tau, h} ;[0, t]\right)$ are bounded independently of $t, \tau$, and $h$. Using the coercivity (3.5) and (3.3) we have found a constant $C>0$ such that for all $\tau, h>0$ the approximants satisfy the bounds

$$
\sup _{t \in[0, T]}\left\|\bar{q}^{\tau, h}(t)\right\|_{\mathrm{H}^{1}(\Omega)} \leq C, \quad\left\|\bar{z}^{\tau, h}\right\|_{\mathrm{BV}\left([0, T], \mathrm{L}^{1}(\Omega)\right)} \leq C, \quad \operatorname{Var}\left(\bar{\eta}^{\tau, h} ;[0, T]\right) \leq C .
$$

Using (P2) of Proposition 3.3 we also have a bound for the power

$$
\forall \tau, h>0 \forall t \in[0, T]:\left|\partial_{t} \mathcal{E}\left(t, \bar{q}^{\tau, h}(t)\right)\right| \leq C .
$$

Step 2. Selection of subsequences. We have prepared all the assumptions for Helly's selection principle as stated in Proposition 5.2. Hence, applying both the classical Helly theorem and Proposition 5.2, we infer that there exists a subsequence $\left(\tau_{n}, h_{n}\right)_{n \in \mathbb{N}}$ such that for all $t \in[0, T]$ we have

$$
\begin{aligned}
& \bar{\eta}^{\tau_{n}, h_{n}}(t) \rightarrow \eta(t), \quad \bar{\delta}^{\tau_{n}, h_{n}}(t) \rightarrow \delta(t), \\
& \bar{z}^{\tau_{n}, h_{n}}(t) \rightarrow z(t) \text { in } \mathcal{Z}, \quad \operatorname{Var}_{\mathcal{D}}(z ;[0, t]) \leq \delta(t)
\end{aligned}
$$

with $\eta \in \mathrm{BV}([0, T] ; \mathbb{R}), \delta:[0, T] \rightarrow \mathbb{R}$ a nondecreasing function, and $z:[0, T] \rightarrow \mathcal{Z}$. 
We want to show that the $u$-component also converges along this subsequence. For this we define

$$
s^{n}(t) \stackrel{\text { def }}{=} \max \left\{t_{j}^{\tau_{n}} \in \Pi^{\tau_{n}} \mid t_{j}^{\tau_{n}} \leq t\right\}
$$

then we have $\bar{q}^{\tau_{n}, h_{n}}(t)=\bar{q}^{\tau_{n}, h_{n}}\left(s^{n}(t)\right) \in \mathcal{S}_{h}\left(s^{n}(t)\right)$. Thus, using $s^{n}(t) \rightarrow t$ and $\bar{z}^{\tau_{n}, h_{n}}(t) \rightarrow z(t)$ we can argue as in Lemma 3.4 to conclude that

$$
\begin{aligned}
& \bar{q}^{\tau_{n}, h_{n}}(t) \rightarrow q(t)=(u(t), z(t)) \text { strongly in } \mathcal{Q}, \\
& \bar{\eta}^{\tau_{n}, h_{n}}(t) \rightarrow \eta(t)=\mathcal{E}(t, q(t)),
\end{aligned}
$$

where $u(t)=\operatorname{Argmin} \mathcal{E}(t, \cdot, z(t))$. Indeed, using the stability of $\bar{q}^{\tau_{n}, h_{n}}(t)$ at $s^{n}(t)$ and testing by $\widehat{q}_{h_{n}} \in \mathcal{Q}_{h_{n}}$ we have

$$
\mathcal{E}\left(s^{n}(t), \bar{q}^{\tau_{n}, h_{n}}(t)\right) \leq \mathcal{E}\left(s^{n}(t), \widehat{q}_{h_{n}}\right)+\mathcal{D}\left(\bar{z}^{\tau_{n}, h_{n}}(t), \widehat{z}_{h_{n}}\right),
$$

and using (3.7)

$$
\begin{aligned}
& \mathcal{E}\left(t, \bar{q}^{\tau_{n}, h_{n}}(t)\right)=\bar{\eta}^{\tau_{n}, h_{n}}(t) \leq \exp \left(C_{1}^{\varepsilon}\left|t-s^{n}(t)\right|\right)\left(\mathcal{E}\left(s^{n}(t), \bar{q}^{\tau_{n}, h_{n}}(t)\right)+C_{0}^{\varepsilon}\right)-C_{0}^{\varepsilon} \\
& \leq \exp \left(C_{1}^{\varepsilon}\left|t-s^{n}(t)\right|\right)\left(\mathcal{E}\left(s^{n}(t), \widehat{q}_{h_{n}}\right)+\mathcal{D}\left(\bar{z}^{\tau_{n}, h_{n}}(t), \widehat{z}_{h_{n}}\right)+C_{0}^{\varepsilon}\right)-C_{0}^{\varepsilon} .
\end{aligned}
$$

By the density assumption (5.1) we may choose $\widehat{q}_{h_{n}}$ such that $\widehat{q}_{h_{n}} \rightarrow q(t)$, and using the strong continuity of $\mathcal{E}$ we obtain $\lim \sup _{n \rightarrow \infty} \bar{\eta}^{\tau_{n}, h_{n}}(t) \leq \mathcal{E}(t, q(t))$. Then we obtain as in Lemma 3.4 that the whole sequence $\left(\bar{q}^{\tau_{n}, h_{n}}(t)\right)_{n \in \mathbb{N}}$ converges weakly to $q(t)$ in $\mathcal{Q}$. By weak lower semicontinuity we again have $\mathcal{E}(t, q(t)) \leq \liminf _{n \rightarrow \infty} \bar{\eta}^{\tau_{n}}, h_{n}(t)$. Thus, the second statement in (5.25) is established. Finally, the strong convergence follows as in Lemma 3.4 by strict convexity.

Now we employ Lemma 3.5 and obtain that the power $\partial_{t} \mathcal{E}\left(t, \bar{q}^{\tau_{n}, h_{n}}(t)\right)$ also converges:

$$
\forall t \in[0, T]: \partial_{t} \mathcal{E}\left(t, \bar{q}^{\tau_{n}, h_{n}}(t)\right) \rightarrow \partial_{t} \mathcal{E}(t, q(t)) .
$$

Step 3. Stability of the limit $q$. To prove that $q(t) \in \mathcal{S}(t)$ we take an arbitrary $\widehat{q} \in \mathcal{Q}$ and have to show $\mathcal{E}(t, q(t)) \leq \mathcal{E}(t, \widehat{q})+\mathcal{D}(z(t), \widehat{z})$. To do so, we apply the density assumption (5.1) and obtain a sequence $\widehat{q}_{n} \in \mathcal{Q}_{h_{n}}$ with $\widehat{q}_{n} \rightarrow \widehat{q}$. Since $\bar{q}^{\tau_{n}, h_{n}}(t) \in$ $\mathcal{S}_{h_{n}}\left(s^{n}(t)\right)$ we have

$$
\mathcal{E}\left(s^{n}(t), \bar{q}^{\tau_{n}, h_{n}}(t)\right) \leq \mathcal{E}\left(s^{n}(t), \widehat{q}_{n}\right)+\mathcal{D}\left(\bar{z}^{\tau_{n}, h_{n}}(t), \widehat{z}_{n}\right) .
$$

Using the convergences $s^{n}(t) \rightarrow t, \bar{q}^{\tau_{n}, h_{n}}(t) \rightarrow q(t)$, and $\widehat{q}_{n} \rightarrow \widehat{q}$, we can pass to the limit and obtain the desired stability.

Step 4. Upper energy estimate. The upper energy estimate on $[0, t]$ follows from the discrete upper energy estimate obtained above for the solutions of the incremental problems. Using (3.7) and (5.14) we find that there exists $C>0$ such that

$$
\mathcal{E}\left(t, \bar{q}^{\tau, h}(t)\right)+\operatorname{Var}_{\mathcal{D}}\left(\bar{z}^{\tau, h} ;[0, t]\right) \leq \eta_{k-1}^{\tau, h}+\operatorname{Var}_{\mathcal{D}}\left(\bar{z}^{\tau, h} ;\left[0, t_{k-1}^{\tau}\right]\right)+C\left(\exp \left(C_{1}^{\mathcal{E}} \tau\right)-1\right)
$$

if $t_{k-1}^{\tau, h} \leq t<t_{k}^{\tau, h}$, which implies thanks to (5.4) that

$$
\bar{\eta}^{\tau, h}(t)+\bar{\delta}^{\tau, h}(t) \leq \eta_{0}^{h}+\int_{0}^{t} \partial_{t} \mathcal{E}\left(s, \bar{q}^{\tau, h}(s)\right) \mathrm{d} s+C\left(\exp \left(C_{1}^{\mathcal{E}} \tau\right)-1\right)
$$


Notice that (5.9) and (5.25) imply that $\lim _{h \rightarrow 0} \eta_{0}^{h}=\mathcal{E}\left(0, q_{0}\right)=\eta(0)$. Then, with (5.23), (5.24), and (5.26), we get at the limit

$$
\mathcal{E}(t, q(t))+\operatorname{Var}_{\mathcal{D}}(z ;[0, t]) \leq \eta(t)+\delta(t) \leq \mathcal{E}\left(0, q_{0}\right)+\int_{0}^{t} \partial_{t} \mathcal{E}(s, q(s)) \mathrm{d} s .
$$

Step 5. Lower energy estimate. Let us prove now the lower energy estimate. As has been observed in [MTL02, MiT04], it will be derived directly from the stability property and not from the discrete lower energy inequality. See also [Mie05, Prop. 5.7] for an abstract result.

With (5.23) we infer that $t \mapsto \partial_{t} \mathcal{E}(t, q(t))$ belongs to $\mathrm{L}^{\infty}([0, T])$. Thus, using a classical result about Lebesgue's integral (see [FrM06]), for each $t \in(0, T]$ there exists a sequence of partitions $\Pi^{n} \stackrel{\text { def }}{=}\left\{0=t_{0}^{n}<t_{1}^{n}<\cdots<t_{N_{n}}^{n}=t\right\}$ of $[0, t]$ with fineness $\Delta\left(\Pi^{n}\right) \stackrel{\text { def }}{=} \max \left\{t_{j}^{n}-t_{j-1}^{n} \mid j=1, \ldots, N_{n}\right\}$ tending to 0, such that

$$
\int_{0}^{t} \partial_{t} \mathcal{E}(s, q(s)) \mathrm{d} s=\lim _{n \rightarrow \infty} \sum_{j=1}^{N_{n}} \partial_{t} \mathcal{E}\left(t_{j}^{n}, q\left(t_{j}^{n}\right)\right)\left(t_{j}^{n}-t_{j-1}^{n}\right) .
$$

For $j \in\left\{1, \ldots, N_{n}\right\}$ we have $q\left(t_{j-1}^{n}\right) \in \mathcal{S}\left(t_{j-1}^{n}\right)$, and by choosing $\bar{q}=q\left(t_{j}^{n}\right)$ we obtain

$$
\begin{aligned}
\mathcal{E}\left(t_{j-1}^{n}, q\left(t_{j-1}^{n}\right)\right) & \leq \mathcal{E}\left(t_{j-1}^{n}, q\left(t_{j}^{n}\right)\right)+\mathcal{D}\left(q\left(t_{j-1}^{n}\right), q\left(t_{j}^{n}\right)\right) \\
& =\mathcal{E}\left(t_{j}^{n}, q\left(t_{j}^{n}\right)\right)+\mathcal{D}\left(q\left(t_{j-1}^{n}\right), q\left(t_{j}^{n}\right)\right)-\int_{t_{j-1}^{n}}^{t_{j}^{n}} \partial_{t} \mathcal{E}\left(s, q\left(t_{j}^{n}\right)\right) \mathrm{d} s .
\end{aligned}
$$

After summation over $j=1, \ldots, N_{n}$, we find

$$
\begin{aligned}
\mathcal{E}(t, q(t))-\mathcal{E}(0, q(0))+\operatorname{Var}_{\mathcal{D}}(q ;[0, t]) & \geq \sum_{j=1}^{N_{n}} \int_{t_{j-1}^{n}}^{t_{j}^{n}} \partial_{t} \mathcal{E}\left(s, q\left(t_{j}^{n}\right)\right) \mathrm{d} s \\
& \geq \sum_{j=1}^{N_{n}} \partial_{t} \mathcal{E}\left(t_{j}^{n}, q\left(t_{j}^{n}\right)\right)\left(t_{j}^{n}-t_{j-1}^{n}\right)+\sum_{j=1}^{N_{n}} \mu_{j}^{n},
\end{aligned}
$$

where $\mu_{j}^{n} \stackrel{\text { def }}{=} \int_{t_{j-1}^{n}}^{t_{j}^{n}}\left(\partial_{t} \mathcal{E}\left(s, q\left(t_{j}^{n}\right)\right)-\partial_{t} \mathcal{E}\left(t_{j}^{n}, q\left(t_{j}^{n}\right)\right)\right) \mathrm{d} s$. Then (P3) implies that there exists a nondecreasing function $\omega^{E}:[0, \infty) \rightarrow[0, \infty)$ with $\omega^{E}(\tau) \rightarrow 0$ for $\tau \searrow 0$ and $E \geq \sup _{t \in[0, T]} c_{0}\|q(t)\|_{\mathcal{Q}}^{2}+c_{0}$ such that

$$
\left|\mu_{j}^{n}\right| \leq\left(t_{j}^{n}-t_{j-1}^{n}\right) \omega^{E}\left(t_{j}^{n}-t_{j-1}^{n}\right) \leq\left(t_{j}^{n}-t_{j-1}^{n}\right) \omega^{E}\left(\Delta\left(\Pi^{n}\right)\right) .
$$

Then passing to the limit in $(5.28)$ as $\Delta\left(\Pi^{n}\right)$ tends to zero, we obtain

$$
\mathcal{E}(t, q(t))-\mathcal{E}(0, q(0))+\operatorname{Var}_{\mathcal{D}}(q ;[0, t]) \geq \int_{0}^{t} \partial_{t} \mathcal{E}(s, q(s)) \mathrm{d} s .
$$

Now, let us recall that $\operatorname{Var}_{\mathcal{D}}(q ;[0, t])=\operatorname{Var}_{\mathcal{D}}(z ;[0, t]) \leq \delta(t)$ with $(5.24)$ and that $\mathcal{E}(t, q(t))=\eta(t)$. Then, the lower and upper energy estimates imply

$$
\begin{aligned}
& \eta(0)+\int_{0}^{t} \partial_{t} \mathcal{E}(s, q(s)) \mathrm{d} s \leq \mathcal{E}(t, q(t))+\operatorname{Var}_{\mathcal{D}}(q ;[0, t]) \\
& \leq \eta(t)+\delta(t) \leq \eta(0)+\int_{0}^{t} \partial_{t} \mathcal{E}(s, q(s)) \mathrm{d} s .
\end{aligned}
$$


Hence, all inequalities are in fact equalities and we deduce that $\delta(t)=\operatorname{Var}_{\mathcal{D}}(q ;[0, t])$. Thus, all assertions in (5.10) are established.

Remark 5.4. Let us observe that, with the choice $\mathcal{F}_{h}=\mathcal{F}$ and $V_{h}=V$ for all $h>0$, we obtain directly the existence of an energetic solution $q=(u, z)$ of $(\mathrm{S})$ and $(\mathrm{E})$.

6. Discussion of stored-energy densities. Here we address some possible stored-energy densities $W: \mathbb{R}_{\mathrm{sym}}^{d \times d} \times Z \times\left[\theta_{\min }, \theta_{\max }\right] \rightarrow \mathbb{R}$ that fulfill the assumptions made in the previous sections. Except for some growth bounds for the different partial derivatives the crucial assumptions are the strict convexity of $W(\cdot, z, \theta): \mathbb{R}_{\mathrm{sym}}^{d \times d} \rightarrow \mathbb{R}$ in $(3.2 \mathrm{a})$ or the much more restrictive assumption of uniform convexity of $W(\cdot, \cdot, \theta)$ : $\mathbb{R}_{\mathrm{sym}}^{d \times d} \times Z \rightarrow \mathbb{R}$ from (4.1).

In [MiT99, Mie00, MTL02] the mixture function $W_{\text {mix }}$ (see (2.4)) was introduced and further analyzed. Using the theory developed in [MTL02, section 4] it follows that starting from convex pure phases $W\left(\cdot, \widehat{e}_{k}, \theta\right)$ the resulting $W_{\text {mix }}(\cdot, z, \theta)$ is still convex for each $z \in Z$. Moreover, if all $W\left(\cdot, \widehat{e}_{k}, \theta\right)$ are quadratic and have the same elastic tensor, then $W_{\text {mix }}$ takes the quadratic form given in (2.5). However, it should be noted that even in this simple case the function $W_{\text {mix }}(\cdot, \cdot, \theta)$ is in general not jointly convex in $(e, z)$. The general theory states that $w_{\operatorname{mix}}(\cdot, \theta): Z \rightarrow \mathbb{R}$ is convex, but the desired convexity of $W_{\text {mix }}(\cdot, \cdot, \theta)$ in $(2.5)$ needs that

$$
\begin{aligned}
\widetilde{w}(\cdot, \theta): z \mapsto & w_{\text {mix }}(z, \theta)+\frac{1}{2}\left(\sum_{k=1}^{N} z_{k} E_{k}(\theta): \mathbb{C}(\theta): E_{k}(\theta)-E(z, \theta): \mathbb{C}(\theta): E(z, \theta)\right) \\
& +\sum_{k=1}^{N} z_{k} w_{k}^{\text {therm }}(\theta)
\end{aligned}
$$

is convex. For $N=2$ convexity holds if and only if $E_{1}$ and $E_{2}$ are symmetrically rank1-connected; see [Mie00, GMH02]. Clearly, we have convexity of $W_{\text {mix }}$ with respect to $e \in \mathbb{R}_{\text {sym }}^{d \times d}$ in all these cases. If additionally $\widetilde{w}$ is uniformly convex in $z$, then also $W_{\text {mix }}$ defined in (2.5) is uniformly convex with respect to $(e, z)$.

In the next lemma, we clarify the assumptions on $w_{\text {mix }}, \mathbb{C}$, and $E$ which imply that $W_{\text {mix }}$ satisfies the assumptions (3.2). Then, we may deduce that all the results given above are also valid in the particular case where $W=W_{\text {mix }}$. The verification is left to the reader.

Lemma 6.1. Assume that $w_{\text {mix }}, \mathbb{C}, E_{j}$, and $w_{j}^{\text {therm }}$ satisfy $w_{\operatorname{mix}}, \partial_{\theta} w_{\operatorname{mix}} \in \mathrm{C}^{0}(Z \times$ $\left.\left[\theta_{\text {min }}, \theta_{\text {max }}\right] ; \mathbb{R}\right), \mathbb{C} \in \mathrm{C}^{1}\left(\left[\theta_{\text {min }}, \theta_{\text {max }}\right] ; \operatorname{Lin}\left(\mathbb{R}_{\text {sym }}^{d \times d} ; \mathbb{R}_{\text {sym }}^{d \times d}\right)\right), E_{j} \in \mathrm{C}^{1}\left(\left[\theta_{\text {min }}, \theta_{\text {max }}\right] ; \mathbb{R}_{\text {sym }}^{d \times d}\right)$, and $w_{j}^{\text {therm }} \in \mathrm{C}^{1}\left(\left[\theta_{\min }, \theta_{\max }\right]\right)$ for $j=1, \ldots, N$. Further assume that there exist $C_{\text {mix }}^{\theta}$ and $\alpha_{\mathbb{C}}>0$ such that for all $z_{1}, z_{2} \in Z, e \in \mathbb{R}_{\mathrm{sym}}^{d \times d}$, and $\theta \in\left[\theta_{\min }, \theta_{\max }\right]$ we have

$$
\left|\partial_{\theta} w_{\text {mix }}\left(z_{1}, \theta\right)-\partial_{\theta} w_{\text {mix }}\left(z_{2}, \theta\right)\right| \leq C_{\text {mix }}^{\theta}\left|z_{1}-z_{2}\right| \text { and } e: \mathbb{C}(\theta): e \geq \alpha_{\mathbb{C}}|e|^{2} .
$$

Then $W_{\text {mix }}$ defined in (2.5) satisfies the assumptions (3.2).

If additionally there exists $\alpha_{\mathrm{mix}}>0$ such that $\widetilde{w}(\cdot, \theta)$ is $\alpha_{\mathrm{mix}}$-uniformly convex for all $\theta \in\left[\theta_{\min }, \theta_{\max }\right]$, then the joint uniform convexity (4.1) for $W=W_{\operatorname{mix}}$ also holds.

In general, it is much too difficult to calculate $W_{\text {mix }}$ explicitly. Hence, it is necessary to model suitably. The general theory in [MTL02] states that $\nabla u \mapsto$ $W_{\text {mix }}(\mathbf{e}(u), z, \theta)$ is always quasi-convex and that $z \mapsto W_{\operatorname{mix}}(e, z, \theta)$ is always convex. Of course, cross-quasi convexity is even stronger but very difficult to characterize. So we need to interpolate between the pure phases $W_{k}(\cdot, \theta)=W\left(\cdot, \widehat{e}_{k}, \theta\right)$ by making 
suitable assumptions. Throughout we assume that each $W_{k}$ is strictly convex with respect to $e$. Here, we are now able to treat much more general functions $W_{k}$. Again we may take an affine interpolation plus a mixture term:

$$
W_{\text {mix }}(e, z, \theta)=\sum_{k=1}^{N} z_{k} W_{k}(e, \theta)+w_{\operatorname{mix}}(z, \theta) \quad \text { for } \quad z=\sum_{k=1}^{N} z_{k} \widehat{e}_{k} .
$$

Clearly, we keep strict convexity with respect to $e$.

In [Mie07] a more general interpolation is suggested in the form

$$
W_{\text {mix }}(e, z, \theta)=\frac{1}{\beta(\theta)} \ln \left(\sum_{k=1}^{N} z_{k} \exp \left(\beta(\theta) W_{k}(e, \theta)\right)\right)+w_{\text {mix }}(z, \theta),
$$

where the limit $\beta \searrow 0$ corresponds to the affine interpolation given in the previous formula. It is simple to see that upper and lower bounds like $c|e|^{2}-C \leq W_{k} \leq C|e|^{2}+C$ directly carry over to $W_{\text {mix }}$. The good message is that also (strict) convexity in $e$ is maintained. To prove this it is sufficient to show that $s \mapsto w(s)=W_{\text {mix }}(e+s \widetilde{e}, z, \theta)$ is convex in $s \in \mathbb{R}$ for all $e, \widetilde{e} \in \mathbb{R}_{\text {sym }}^{d \times d}$. We let $w_{k}(s)=W_{k}(e+s \widetilde{e}, \theta)$ and assume, for simplicity, that all $w_{k}$ are twice differentiable; then we obtain

$$
\begin{aligned}
w^{\prime \prime}= & \exp \left(-\beta\left(w-w_{\text {mix }}\right)\right) \sum_{k=1}^{N} z_{k} \exp \left(\beta w_{k}\right) w_{k}^{\prime \prime}+\beta \exp \left(-2 \beta\left(w-w_{\text {mix }}\right)\right) \\
& \left(\left(\sum_{k=1}^{N} z_{k} \exp \left(\beta w_{k}\right)\right)\left(\sum_{k=1}^{N} z_{k} \exp \left(\beta w_{k}\right)\left(w_{k}^{\prime}\right)^{2}\right)-\left(\sum_{k=1}^{N} z_{k} \exp \left(\beta w_{k}\right) w_{k}^{\prime}\right)^{2}\right) .
\end{aligned}
$$

Obviously the first sum is nonnegative, and the last term in big parentheses is nonnegative as well by a simple application of the Cauchy-Schwarz inequality.

The above discussion shows that there is a wide variety of possible mixture functions $W_{\text {mix }}: \mathbb{R}_{\text {sym }}^{d \times d} \times Z \times\left[\theta_{\text {min }}, \theta_{\text {max }}\right] \rightarrow \mathbb{R}$ for which the above existence theory is applicable.

Acknowledgment. L. P. wishes to thank WIAS for its kind hospitality as well as the friendly atmosphere there.

\section{REFERENCES}

[AGR03] M. ARndt, M. Griebel, And T. RoubìčEK, Modelling and numerical simulation of martensitic transformation in shape memory alloys, Contin. Mech. Thermodyn., 15 (2003), pp. 463-485.

[AmS08] F. Auricchio, A. Mielke, And U. Stefanelli, A rate-independent model for the isothermal quasi-static evolution of shape-memory materials, Math. Models Methods Appl. Sci., 18 (2008), pp. 125-164.

[AuP04] F. Auricchio AND L. PETRINI, A three-dimensional model describing stress-temperature induced solid phase transformations. Part II: Thermomechanical coupling and hybrid composite applications, Internat. J. Numer. Methods Engrg., 61 (2004), pp. 716-737.

[AuS01] F. AURICCHIO AND E. SACCO, Thermo-mechanical modelling of a superelastic shapememory wire under cyclic stretching-bending loadings, Internat. J. Solids Structures, 38 (2001), pp. 6123-6145.

[AuS04] F. Auricchio AND U. StefAnElli, Numerical treatment of a $3 D$ super-elastic constitutive model, Internat. J. Numer. Meth. Engrg., 61 (2004), pp. 142-155. 
[AuS05] F. Auricchio AND U. Stefanelli, Well-posedness and approximation for a onedimensional model for shape memory alloys, Math. Models Methods Appl. Sci., 15 (2005), pp. 1301-1327.

[CaP01] C. CARstensen And P. Plecháč, Numerical analysis of a relaxed variational model of hysteresis in two-phase solids, M2AN Math. Model. Numer. Anal., 35 (2001), pp. 865878.

[DuL76] G. Duvaut And J.-L. Lions, Inequalities in Mechanics and Physics, Springer-Verlag, Berlin, 1976.

[FrM06] G. FRANCFORT AND A. Mielke, Existence results for a class of rate-independent material models with nonconvex elastic energies, J. Reine Angew. Math., 595 (2006), pp. 5591.

[GHH07] S. Govindjee, K. Hackl, And R. Heinen, An upper bound to the free energy of mixing by twin-compatible lamination for $n$-variant martensitic phase transformations, Contin. Mech. Thermodyn., 18 (2007), pp. 443-453.

[GMH02] S. Govindjee, A. Mielke, ANd G. HAll, The free energy of mixing for n-variant martensitic phase transformations using quasi-convex analysis, J. Mech. Physics Solids, 50 (2002), pp. 1897-1922; erratum and correct reprinting: 51 (2003), pp. 763 and IXXVI.

[HaG02] G. Hall And S. Govindjee, Application of the relaxed free energy of mixing to problems in shape memory alloy simulation, J. Intell. Mater. Syst. Struct., 13 (2002), pp. 773782.

[KMR05] M. KRuŽíK, A. Mielke, And T. RoubíČEK, Modelling of microstructure and its evolution in shape-memory-alloy single-crystals, in particular in CuAlNi, Meccanica, 40 (2005), pp. 389-418.

[KoO88] V. Kondrat'Ev And O. Oleinik, Boundary-value problems for the system of elasticity theory in unbounded domains. Korn's inequalities, Russian Math. Surveys, 43 (1988), pp. 65-119.

[MaM05] A. MAINIK AND A. Mielke, Existence results for energetic models for rate-independent systems, Calc. Var. Partial Differential Equations, 22 (2005), pp. 73-99.

[Mie00] A. Mielke, Estimates on the mixture function for multiphase problems in elasticity, in Multifield Problems, A.-M. Sändig, W. Schiehlen, and W. Wendland, eds., SpringerVerlag, Berlin, 2000, pp. 96-103.

[Mie05] A. Mielke, Evolution in rate-independent systems, in Handbook of Differential Equations, Evolutionary Equations, Vol. 2, C. Dafermos and E. Feireisl, eds., Elsevier, Amsterdam, 2005, pp. 461-559.

[Mie07] A. Mielke, A model for temperature-induced phase transformations in finite-strain elasticity, IMA J. Appl. Math., 72 (2007), pp. 644-658.

[MiP07] A. Mielke And A. Petrov, Thermally driven phase transformation in shape-memory alloys, Adv. Math. Sci. Appl., 17 (2007), pp. 667-685.

[MiR06] A. Mielke And T. RoubÍČEK, Numerical approaches to rate-independent processes and applications in inelasticity, M2AN Math. Model. Numer. Anal., 43 (2009), pp. 399428.

[MiR07] A. Mielke AND R. Rossi, Existence and uniqueness results for a class of rate-independent hysteresis problems, Math. Models Methods Appl. Sci., 17 (2007), pp. 81-123.

[MiT99] A. Mielke AND F. Theil, A mathematical model for rate-independent phase transformations with hysteresis, in Proceedings of the Workshop on Models of Continuum Mechanics in Analysis and Engineering, H.-D. Alber, R. Balean, and R. Farwig, eds., Shaker-Verlag, Aachen, 1999, pp. 117-129.

[MiT04] A. Mielke AND F. Theil, On rate-independent hysteresis models, NoDEA Nonlinear Differential Equations Appl., 11 (2004), pp. 151-189.

[MRS08] A. Mielke, T. Roubíček, and U. Stefanelli, $\Gamma$-limits and relaxations for rateindependent evolutionary problems, Calc. Var. Partial Differential Equations, 31 (2008), pp. 387-416.

[MTL02] A. Mielke, F. Theil, And V. I. Levitas, A variational formulation of rate-independent phase transformations using an extremum principle, Arch. Ration. Mech. Anal., 162 (2002), pp. 137-177.

[RaT83] P.-A. RAVIART AND J.-M. Thomas, Introduction à l'analyse numérique des équations aux dérivées partielles. Collection Mathématiques Appliquées pour la Maîtrise, Masson, Paris, 1983.

[RoK06] T. RoubíčEK AND M. KRUŽÍK, Mesoscopic model of microstructure evolution in shape memory alloys, its numerical analysis and computer implementation, GAMM-Mitt., 29 (2006), pp. 192-214. 
[Rou02] T. RoubíčEK, Evolution model for martensitic phase transformation in shape-memory alloys, Interfaces Free Bound., 4 (2002), pp. 111-136.

[Rou04] T. RouBíčEK, Models of microstructure evolution in shape memory alloys, in Nonlinear Homogenization and its Applications to Composites, Polycrystals and Smart Materials, P. Ponte Castaneda, J. Telega, and B. Gambin, eds., Kluwer Academic Publishers, Dordrecht, The Netherlands, 2004, pp. 269-304.

[SMZ98] A. Souza, E. MamiYa, And N. Zouain, Three-dimensional model for solids undergoing stress-induced phase transformations, Eur. J. Mech. A Solids, 17 (1998), pp. 789-806.

[Vis84] A. Visintin, Strong convergence results related to strict convexity, Comm. Partial Differential Equations, 9 (1984), pp. 439-466. 